\title{
1 Unifying Parsing and Reflective Printing for Fully 2 Disambiguated Grammars
}

\author{
3 Zirun Zhu ${ }^{1}$ (1) $\cdot$ Hsiang-Shang $\mathrm{Ko}^{2}$ (D) $\cdot$ Yongzhe Zhang $^{1}$ (D) $\cdot$ Pedro Martins ${ }^{3}$.

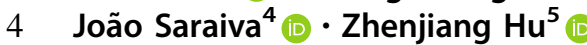

5 Received: 31 January 2019/Accepted: 16 December 2019

6 (C) Ohmsha, Ltd. and Springer Japan KK, part of Springer Nature 2020

\section{Abstract}

8 Language designers usually need to implement parsers and printers. Despite being 9 two closely related programs, in practice they are often designed separately, and 10 then need to be revised and kept consistent as the language evolves. It will be more convenient if the parser and printer can be unified and developed in a single program, with their consistency guaranteed automatically. Furthermore, in certain scenarios (like showing compiler optimisation results to the programmer), it is desirable to have a more powerful reflective printer that, when an abstract syntax tree corresponding to a piece of program text is modified, can propagate the modification to the program text while preserving layouts, comments, and syntactic sugar. To address these needs, we propose a domain-specific language BIYACC, whose programs denote both a parser and a reflective printer for a fully disambiguated context-free grammar. BIYACC is based on the theory of bidirectional transformations, which helps to guarantee by construction that the generated pairs of parsers and reflective printers are consistent. Handling grammatical ambiguity is particularly challenging: we propose an approach based on generalised parsing and disambiguation filters, which produce all the parse results and (try to) select the only correct one in the parsing direction; the filters are carefully bidirectionalised so that they also work in the printing direction and do not break the consistency between the parsers and reflective printers. We show that BIYACC is capable of facilitating many tasks such as Pombrio and Krishnamurthi's 'resugaring', simple refactoring,

Keywords Asymmetric lenses · Disambiguation filters · Bidirectional transformations · Domain-specific languages · Parsing · Reflective printing

Electronic supplementary material The online version of this article (https://doi.org/10.1007/s00354019-00082-y) contains supplementary material, which is available to authorized users.

Extended author information available on the last page of the article

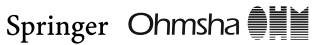

\begin{tabular}{lllll} 
Journal : Small-ext 354 & Dispatch : & $\mathbf{1 3 - 2 - 2 0 2 0}$ & \multicolumn{2}{l}{ Pages : $\mathbf{5 5}$} \\
Article No. $: \mathbf{8 2}$ & $\square \quad$ LE & & $\square$ & TYPESET \\
MS Code : NGCO-D-19-00023R2 & $\square$ & CP & $\square$ & DISK \\
\hline
\end{tabular}




\section{Introduction}

Whenever we come up with a new programming language, as the front-end part of the system we need to design and implement a parser and a printer to convert between program text and an internal representation. A piece of program text, while conforming to a concrete syntax specification, is a flat string that can be easily edited by the programmer. The parser extracts the tree structure from such a string to a concrete syntax tree (CST), and converts it to an abstract syntax tree (AST), which is a more structured and simplified representation and is easier for the backend to manipulate. On the other hand, a printer converts an AST back to a piece of program text, which can be understood by the user of the system; this is useful for debugging the system, or reporting internal information to the user.

Parsers and printers do conversions in opposite directions and are closely related - for example, the program text printed from an AST should be parsed to the same tree. It is certainly far from being economical to write parsers and printers separately: the parser and printer need to be revised from time to time as the language evolves, and each time we must revise the parser and printer and also keep them consistent with each other, which is a time-consuming and error-prone task. In response to this problem, many domain-specific languages [6, 7, 13, 37, 44, 53] have been proposed, in which the user can describe both a parser and a printer in a single program.

Despite their advantages, these domain-specific languages cannot deal with synchronisation between program text and ASTs. Let us look at a concrete example in Fig. 1: the original program text is an arithmetic expression, containing a negation, a comment, and parentheses (one pair of which is redundant). It is first parsed to an AST (supposing that addition is left-associative) where the negation is desugared to a subtraction, parentheses are implicitly represented by the tree structure, and the comment is thrown away. Suppose that the AST is optimised by replacing Add (Num 1) (Num 1) with a constant Num 2. The user may want to observe the optimisation made by the compiler, but the AST is an internal representation not exposed to the user, so a natural idea is to propagate the changes on the AST back to the program text to make it easy for the user to check where the changes are. With a conventional printer, however, the printed result will likely mislead the programmer into thinking that the negation is replaced by a subtraction by the compiler; also, since the comment is not preserved, it will be harder for the

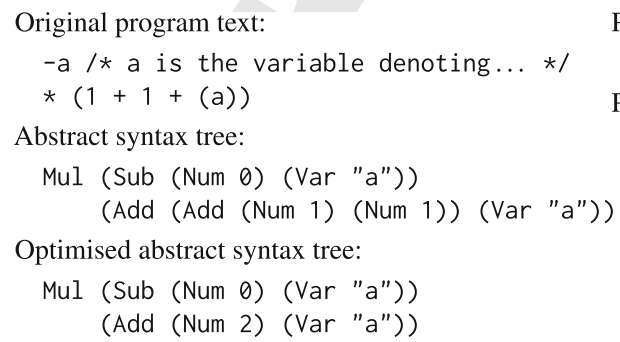

Fig. 1 Comparison between conventional printing and reflective printing

\section{Springer Ohmsha 峷高咅}

\begin{tabular}{|l|lllll|}
\hline & Journal : Small-ext 354 & Dispatch : $\mathbf{1 3 - 2 - 2 0 2 0}$ & Pages : 55 \\
& Article No. : 82 & $\square$ & LE & & TYPESET \\
& MS Code : NGCO-D-19-00023R2 & $\nabla$ & CP & $\square$ & DISK \\
\hline
\end{tabular}


programmer to compare the updated and original versions of the text. The problem illustrated here has also been investigated in many other practical scenarios where the parser and printer are used as a bridge between the system and the user, for example,

- in bug reporting [51], where a piece of program text is parsed to its AST to be checked but error messages should be displayed for the program text;

- in code refactoring [18], where instead of directly modifying a piece of program text, most refactoring tools will first parse the program text into its AST, perform code refactoring on the AST, and regenerate new program text; and

- in language-based editors, as introduced by Reps [45, 46], where the user needs to interact with different printed representations of the same underlying AST.

To address the problem, we propose a domain-specific language BIYACC, which enables the user to describe both a parser and a reflective printer for a fully disambiguated context-free grammar (CFG) in a single program. Different from a conventional printer, a reflective printer takes a piece of program text and an AST, which is usually slightly modified from the AST corresponding to the original program text, and propagates the modification back to the program text. Meanwhile the comments (and layouts) in the unmodified parts of the program text are all preserved. This can be seen clearly from the result of using our reflective printer on the above arithmetic expression example in Fig. 1. It is worth noting that reflective printing is a generalisation of the conventional notion of printing, because a reflective printer can accept an AST and an empty piece of program text, in which case it will behave just like a conventional printer, producing a new piece of program text depending on the AST only.

From a BIYACC program, we can generate a parser and a reflective printer; in addition, we want to guarantee that the two generated components are consistent with each other. Specifically, given a pair of parser parse and reflective printer print, we want to ensure two (inverse-like) consistency properties: first, a piece of program text $s$ printed from an abstract syntax tree $t$ should be parsed to the same tree $t$, i.e. ${ }^{1}$

$$
\text { parse }(\text { print } s t)=t \text {. }
$$

Second, updating a piece of program text $s$ with an AST parsed from $s$ should leave $s$ unmodified (including formatting details like parentheses and whitespaces), i.e.

$$
\text { print } s(\text { parse } s)=s \text {. }
$$

1 We assume basic knowledge about functional programming languages and their notations, in particular HASKELl [5, 34]. In HASKELl, an argument of function application does not need to be enclosed in (round) parentheses, i.e. we write $f x$ instead of $f(x)$; type variables are implicitly universally quantified, i.e. $f:: a \rightarrow b \rightarrow a$ is the same as $f:: \forall a b . a \rightarrow b \rightarrow a$ where $::$ means has type. Additionally, we omit universal quantification for free variables in an equation; for instance, parse (print $s t)=t$ is in fact $\forall s t$. parse (print $s t)=t$.
}

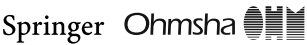

\begin{tabular}{lllll} 
Journal : Small-ext 354 & Dispatch : & $\mathbf{1 3 - 2 - 2 0 2 0}$ & \multicolumn{2}{l}{ Pages : $\mathbf{5 5}$} \\
Article No. $: \mathbf{8 2}$ & $\square \quad$ LE & & $\square$ & TYPESET \\
MS Code : NGCO-D-19-00023R2 & $\square$ & CP & $\square$ & DISK \\
\hline
\end{tabular}


These two properties are inspired by the theory of bidirectional transformations [19], in particular lenses [17], and are guaranteed by construction for all BIYACC programs.

An online tool that implements the approach described in the paper can be accessed at http://www.prg.nii.ac.jp/project/biyacc.html. The webpage also contains the test cases used in the paper. The structure of the paper is as follows: we start with an overview of BIYACC in Sect. 2, explaining how to describe in a single program both a parser and a reflective printer for synchronising program text and its abstract syntax representation. After reviewing some background on bidirectional transformations in Sect. 3, in particular the bidirectional programming language BIGUL [22, 27, 28], we first give the semantics of a basic version of BIYACC that handles unambiguous grammars by compiling it to BIGUL in Sect. 4, guaranteeing the properties (1) and (2) by construction. Then, inspired by the research on generalised parsing [50] and disambiguation filters [26], in Sect. 5 we revise the basic BIYACC architecture to allow the use of ambiguous grammars and disambiguation directives while still retaining the above-mentioned properties. We present a case study in Sect. 6, showing that BIYACC is capable of describing Tiger [4], which shares many similarities with fully fledged languages. We demonstrate that BIYACC can handle syntactic sugar, partially subsume Pombrio and Krishnamurthi's 'resugaring' [42, 43], and facilitate language evolution. In Sect. 7, we present detailed related work including comparison with other systems. Contributions are summarised in Sect. 8.

This is the extended version of our previous work Parsing and Reflective Printing, Bidirectionally presented at SLE' 16 [55], and the differences are mainly as follows: (1) we propose the notion of bidirectionalised filters and integrate them into BIYACC for handling grammatical ambiguity (Sect. 5); the related work section is also updated accordingly. (2) We restructure the narration for introducing the basic BIYACC system and in particular elaborate on the isomorphism between program text and CSTs. (3) We present the definitions and theorems in a more formal way, and complete their proofs. (4) We make several other revisions such as renewing the figures for introducing the BIYACC system and the syntax of BIYACC programs.

Throughout this paper, we typeset general definitions and properties in math style and specific examples in code style.

\section{A First Look at BIYACC}

We first give an overview of BIYACC by going through the BIYACC program shown in Fig. 2, which deals with the arithmetic expression example given in Sect. 1. This program consists of definitions of the abstract syntax, concrete syntax, directives, and actions for reflectively printing ASTs to CSTs; we will introduce them in order.

\section{Syntax Definitions}

Abstract syntax The abstract syntax part, which starts with the keyword \#Abstract, is just one or more definitions of HASKell data types. In our example, the abstract

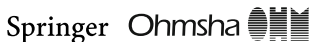

\begin{tabular}{|l|llll|}
\hline & Journal : Small-ext 354 & Dispatch : & $\mathbf{1 3 - 2 - 2 0 2 0}$ & Pages : $\mathbf{5 5}$ \\
Article No. : 82 & $\square$ LE & $\square$ TYPESET \\
MS Code : NGCO-D-19-00023R2 & $\square$ CP & $\square$ & DISK \\
\hline
\end{tabular}


167 The main part of a BIYACC program starts with the keyword \#Actions and describes 168 how to update a CST with an AST. For our expression example, the actions are defined in lines 27-42 in Fig. 2. Before explaining the actions, we should first say that program text is identified with CSTs when programming BIYACC actions: conceptually, whenever we write a piece of program text, we are actually describing a CST rather than just a sequence of characters. We will expound on this identification of program text with CSTs in Sect. 4.2 in detail.

The \#Actions part consists of groups of actions, and each group begins with a 'type declaration' of the form HsType ' $+>$ ' Nonterminal stating that the actions in this group specify updates on CSTs generated from Nonterminal using ASTs of type HsType. Informally, given an AST and a CST, the semantics of an action is to perform pattern matching simultaneously on both trees, and then use components of the AST to update corresponding parts of the CST, possibly recursively. (The syntax ' $+>$ ' suggests that information from the left-hand side is embedded into the right-hand side.) Usually, the nonterminals in a right-hand side pattern are overlaid with updated instructions, which are also denoted by ' $+>$ '.

Let us look at a specific action-the first one for the expression example, at line 28 of Fig. 2:

$$
\text { Add } \mathrm{x} y+>[\mathrm{x}+>\text { Expr }] \text { ' }+ \text { ' }[\mathrm{y}+>\text { Term }] \text {; }
$$

186

187

188

189

190

191

192

193

194

195

196

197

198

199

200

201

202

203

204

205

206

207

208

The AST-side pattern Add $\mathrm{x} y$ is just a HaSkELL pattern; as for the CST-side pattern, the main intention is to refer to the production rule Expr $\rightarrow$ Expr ' + ' Term and use it to match those CSTs produced by this rule-since the action belongs to the group Arith $+>$ Expr, the part 'Expr $\rightarrow$ ' of the production rule can be inferred and thus is not included in the CST-side pattern. Finally, we overlay ' $x+>$ ' and ' $y+>$ ' on the nonterminal symbols Expr and Term to indicate that, after the simultaneous pattern matching succeeds, the subtrees $x$ and $y$ of the AST are, respectively, used to update the left and right subtrees of the CST.

Having explained what an action means, we can now explain the semantics of the entire program. Given an AST and a CST as input, first a group (of actions) is chosen according to the types of the trees. Then, the actions in the group are tried in order, from top to bottom, by performing simultaneous pattern matching on both trees. If pattern matching for an action succeeds, the updating operations specified by the action is executed, otherwise the next action is tried. Execution of the program ends when the matched action specifies either no updating operations or only updates to primitive data types such as Numeric. BIYACC's most interesting behaviour shows up when all actions in the chosen group fail to match-in this case a suitable CST will be created. The specific approach adopted by BIYACC is to perform pattern matching on the AST only and choose the first matched action. A suitable CST conforming to the CST-side pattern is then created, and after that the whole group of actions is tried again. This time the pattern matching will succeed at the action used to create the CST, and the program will be able to make further progress. For instance, assuming that the source is $1{ }^{*} 2$ while the view is Add (Num

\section{Springer Ohmsha 峷基}

\begin{tabular}{|l|lllll|}
\hline & Journal : Small-ext 354 & Dispatch : 13-2-2020 & Pages : 55 \\
Article No. : 82 & $\square$ LE & $\square$ & TYPESET \\
& MS Code : NGCO-D-19-00023R2 & $\square$ CP & $\square$ DISK \\
\hline
\end{tabular}


1) (Num 2), a new source skeleton representing ${ }_{-}+{ }_{-}$will be created and the - part will be updated recursively later. We will elaborate more on this in Sect. 4.

Deep patterns Using deep patterns, we can write actions that establish nontrivial relationships between CSTs and ASTs. For example, the action at line 38 of Fig. 2 associates abstract subtraction expressions whose left operand is zero with concrete negated expressions; this action is the key to preserving negated expressions in the CST. For an example of a more complex CST-side pattern: suppose that we want to write a pattern that matches those CSTs produced by the rule Factor $\rightarrow$ ' '-' Factor, where the inner nonterminal Factor produces a further '-' Factor using the same rule. This pattern is written by overlaying the production rule on the first nonterminal Factor (an additional pair of parentheses is required for the expanded nonterminal): '-' (Factor $\rightarrow>$ '-' Factor). More examples involving this kind of deep patterns can be found in Sect. 6 .

Layout and comment preservation The reflective printer generated by $\mathrm{BI}_{\mathrm{I}} \mathrm{ACC}$ is capable of preserving layouts and comments, but, perhaps mysteriously, in Fig. 2 there is no clue as to how layouts and comments are preserved. This is because we decide to hide layout preservation from the user, so that the more important logic of abstract and concrete syntax synchronisation is not cluttered with layout preserving instructions. Our approach is fairly simplistic: we store layout information following each terminal in an additional field in the CST implicitly, and treat comments in the same way as layouts. During the printing stage, if the pattern matching on an action succeeds, the layouts and comments after the terminals shown in the right-hand side of that action are preserved; on the other hand, layouts and comments are dropped when a CST is created in the situation where pattern matching fails for all actions in a group. The layouts and comments before the first terminal are always kept during the printing.

Parsing semantics So far, we have been describing the reflective printing semantics of the BIYACC program, but we may also work out its parsing semantics intuitively by interpreting the actions from right to left, converting the production rules to the corresponding constructors. (This might remind the reader of the usual YACC [23] actions.) In fact, this paper will not define the parsing semantics formally, because the parsing semantics is completely determined by the reflective printing semantics: if the actions are written with the intention of establishing some relation between the CSTs and ASTs, then BIYACC will be able to derive the only wellbehaved parser, which respects that relation. We will explain how this is achieved in the next section.

\section{Foundation of BIYACC: Putback-Based Bidirectional Programming}

From a BIYACC program, in addition to generating a parser and a printer, we also need to guarantee that the two generated programs are consistent with each other, i.e. satisfy the properties (1) and (2) stated in Sect. 1. It is possible to implement the print and parse semantics separately in an ad hoc way, but verifying the two consistency properties takes extra effort. The implementation we present, however, is systematic and guarantees consistency by construction, thanks to the well-

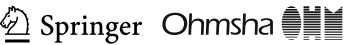

\begin{tabular}{lllll} 
Journal : Small-ext 354 & Dispatch : & $\mathbf{1 3 - 2 - 2 0 2 0}$ & \multicolumn{2}{c}{ Pages : $\mathbf{5 5}$} \\
Article No. $: \mathbf{8 2}$ & $\square$ LE & & $\square$ & TYPESET \\
MS Code : NGCO-D-19-00023R2 & $\square$ & CP & $\square$ DISK \\
\hline
\end{tabular}


developed theory of bidirectional transformations (BXs for short), in particular lenses [17]. We will give a brief introduction to BXs below; for a comprehensive treatment, the readers are referred to the lecture notes for the 2016 Oxford Summer School on Bidirectional Transformations [19].

\section{Parsing and Printing as Lenses}

257

258

259

260

261

262

263

264

The parse and print semantics of BIYACC programs are potentially partial-for example, if the actions in a BIYACC program do not cover all possible forms of program text and abstract syntax trees, parse and print will fail for those uncovered inputs. Thus, we should take partiality into account when choosing a BX framework in which to model parse and print. The framework we use in this paper is an explicitly partial version [32, 40] of asymmetric lenses [17].

Definition 1 (Lenses) A lens between a source type $S$ and a view type $V$ is a pair of functions

$$
\begin{aligned}
& \text { get }:: S \rightarrow \text { Maybe } V \\
& \text { put }:: S \rightarrow V \rightarrow \text { Maybe } S
\end{aligned}
$$

satisfying the well-behavedness laws:

$$
\begin{aligned}
& \text { put } s v=\text { Just } s^{\prime} \Rightarrow \text { get } s^{\prime}=\text { Just } v \quad \text { (PutGet) } \\
& \text { get } s=\text { Just } v \Rightarrow \text { put } s v=\text { Just } s \quad \text { (GeтPut) }
\end{aligned}
$$

Intuitively, a get function extracts a part of a source of interest to the user as a view, and a put function takes a source and a view and produces an updated source incorporating information from the view. Partiality is explicitly represented by making the functions return Maybe values: a get or put function returns Just $r$ where $r$ is the result, or Nothing if the input is not in the domain. The PutGet law enforces that put must embed all information of the view into the updated source, so the view can be recovered from the source by get, while the GeтPut law prohibits put from performing unnecessary updates by requiring that putting back a view directly extracted from a source by get must produce the same, unmodified source.

The parse and print semantics of a BIYACC program will be the pair of functions get and put in a lens, required by definition to satisfy the two well-behavedness laws, which are exactly the consistency properties (1) and (2) reformulated in a partial setting:

Definition 2 (The Partial Version of Consistency Properties)

$$
\begin{aligned}
\text { print } s t=\text { Just } s^{\prime} & \Rightarrow \text { parse } s^{\prime}=\text { Just } t \\
\text { parse } s=\text { Just } t & \Rightarrow \quad \text { print } s t=\text { Just } s
\end{aligned}
$$

\begin{tabular}{|l|lllll|}
\hline & Journal : Small-ext 354 & Dispatch : 13-2-2020 & Pages : 55 \\
& Article No. : 82 & $\square$ & LE & & TYPESET \\
& MS Code : NGCO-D-19-00023R2 & $\nabla$ & CP & & DISK \\
\hline
\end{tabular}


285

286

287

288

289

290

291

292

293

294

295

296

297

298

299

300

301

302

303

304

305

306

308

309

310

311

312

Having rephrased parsing and printing in terms of lenses, we can now construct consistent pairs of parsers and printers using bidirectional programming techniques, in which the programmer writes a single program to denote the two directions of a lens. Specifically, BIYACC programs are compiled to the putback-based bidirectional programming language BiGUL [28]. It has been formally verified in Agda [39] that BIGUL programs always denote well-behaved lenses, and BIGUL has been ported to HASKELL as an embedded DSL library [22]. BiGUL is putback-based, meaning that a BiGUL program describes a put function, but-since BIGUL is bidirectional — can also be executed as the corresponding get function. The advantage of putback-based bidirectional programming lies in the fact that, given a put function, there is at most one get function that forms a (well-behaved) lens with this put function [16]. That is, once we describe a put function as a BIGUL program, the get semantics of the program is completely determined by its put semantics. We can therefore focus solely on the printing (put) behaviour, leaving the parsing (get) behaviour only implicitly (but unambiguously) specified. How the programmer can effectively work with this paradigm has been more formally explained in terms of a Hoare-style logic for BIGUL [27].

Compilation of BIYACC to BiGUL (Sect. 4) uses only three BIGUL operations, which we briefly introduce here; more details can be found in the lecture notes on BiGUL programming [22]. A BiGUL program has type BiGUL $s v$, where $s$ and $v$ are, respectively, the source and view types.

Replace The simplest BiGUL operation we use is

\section{Replace :: BiGUL s s}

which discards the original source and returns the view-which has the same type as the source-as the updated source. That is, the put semantics of Replace is the function $\lambda s v \rightarrow$ Just $v$.

Update The next operation update is more complex, and is implemented with the help of Template Haskell [49]. The general form of the operation is

$$
\$ \text { (update }[p \mid \text { spat } \mid][p|v p a t|][d|b s|]):: \text { BiGUL } s v \text {. }
$$

This operation decomposes the source and view by pattern matching with the patterns spat and vpat, respectively, pairs the source and view components as specified by the patterns (see below), and performs further BIGUL operations listed in $b s$ on the source-view pairs; the way to determine which source and view components are paired and which operation is performed on a pair is by looking for the same names in the three arguments. For example, the update operation

$$
\$(\text { update }[\mathrm{p}|(\mathbf{x},-)|][\mathrm{p}|\mathbf{x}|][\mathrm{d} \mid \mathrm{x}=\text { Replace } \mid])
$$

matches the source with a tuple pattern $\left(\mathbf{x},_{-}\right)$and the view with a variable pattern $\mathbf{x}$, so that the first component of the source tuple is related with the whole view; during the update, the first component of the source is replaced by the whole view, as indicated by the operation $x=$ Replace. (The part marked by underscore (_) simply

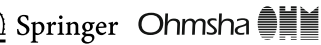

\begin{tabular}{lllll} 
Journal : Small-ext 354 & Dispatch : & $\mathbf{1 3 - 2 - 2 0 2 0}$ & \multicolumn{2}{c}{ Pages : $\mathbf{5 5}$} \\
Article No. $: \mathbf{8 2}$ & $\square$ LE & & $\square$ & TYPESET \\
MS Code : NGCO-D-19-00023R2 & $\square$ & CP & $\square$ DISK \\
\hline
\end{tabular}


means that it will be skipped during the update.) Given a source $(1,2)$ and a view 3 , the operation will produce $(3,2)$ as the updated source. In general, any (type-correct) BiGUL program can be used in the list of further updates, not just the primitive Replace.

Case The most complex operation we use is Case for doing case analysis on the source and view:

$$
\text { Case }::[\text { Branch } s v] \rightarrow \text { BiGUL } s v \text {. }
$$

Case takes a list of branches, of which there are two kinds: normal branches and adaptive branches. For a normal branch, we should specify a main condition using a source pattern spat and a view pattern vpat, and an exit condition using a source pattern spat':

$$
\$(\text { normalSV }[p \mid \text { spat } \mid][p \mid \text { vpat } \mid][p \mid \text { spat } \mid]):: \text { BiGUL } s v \rightarrow \text { Branch } s v .
$$

An adaptive branch, on the other hand, only needs a main condition:

$$
\$(\text { adaptiveSV }[p \mid \text { spat } \mid][p \mid \text { vpat } \mid])::(s \rightarrow v \rightarrow s) \rightarrow \text { BiGUL } s v .
$$

Their semantics in the put direction are as follows: a branch is applicable when the source and view, respectively, match spat and vpat in its main condition. Execution of a Case chooses the first applicable branch from the list of branches, and continues with that branch. When the applicable branch is a normal branch, the associated BIGUL operation is performed, and the updated source should satisfy the exit condition spat (or otherwise execution fails); when the applicable branch is an adaptive branch, the associated function is applied to the source and view to compute an adapted source, and the whole Case is rerun on the adapted source and the view; it must go into a normal branch this time, otherwise the execution fails. Think of an adaptive branch as bringing a source that is too mismatched with the view to a suitable shape-for example, when the source is a subtraction while the view is an addition, which are by no means in correspondence, we must adapt the source to an addition - so that a normal branch that deals with sources and views in some sort of correspondence can take over. This adaptation mechanism is used by BIYACC to print an AST when the source program text is too different from the AST or even nonexistent at all.

\section{The Basic BIYACC}

In this section, we expound on a basic version of BIYACC that handles only unambiguous grammars. (Section 5 will present extensions for dealing with ambiguous grammars with disambiguation.) The architecture is illustrated in Fig. 3, where a BIYACC program

$$
\text { '\#Abstract' decls '\#Concrete' pgs '\#Directives' drctvs '\#Actions' ags, }
$$

consisting of abstract syntax, concrete syntax, directives, and printing actions, as formally defined in Fig. 4, is compiled into a few HASKELl source files and then into

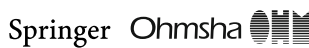

\begin{tabular}{|l|lllll|}
\hline & Journal : Small-ext 354 & Dispatch : 13-2-2020 & Pages : 55 \\
& Article No. : 82 & $\square$ LE & & TYPESET \\
& MS Code : NGCO-D-19-00023R2 & $\square$ CP & $\square$ & DISK \\
\hline
\end{tabular}




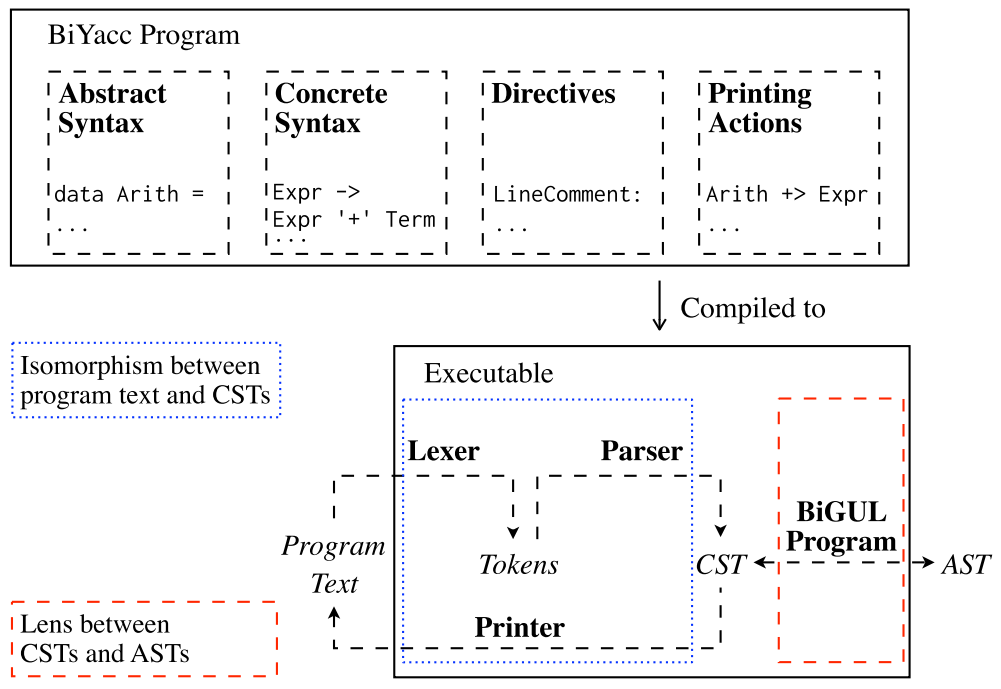

Fig. 3 Architecture of BIYACC

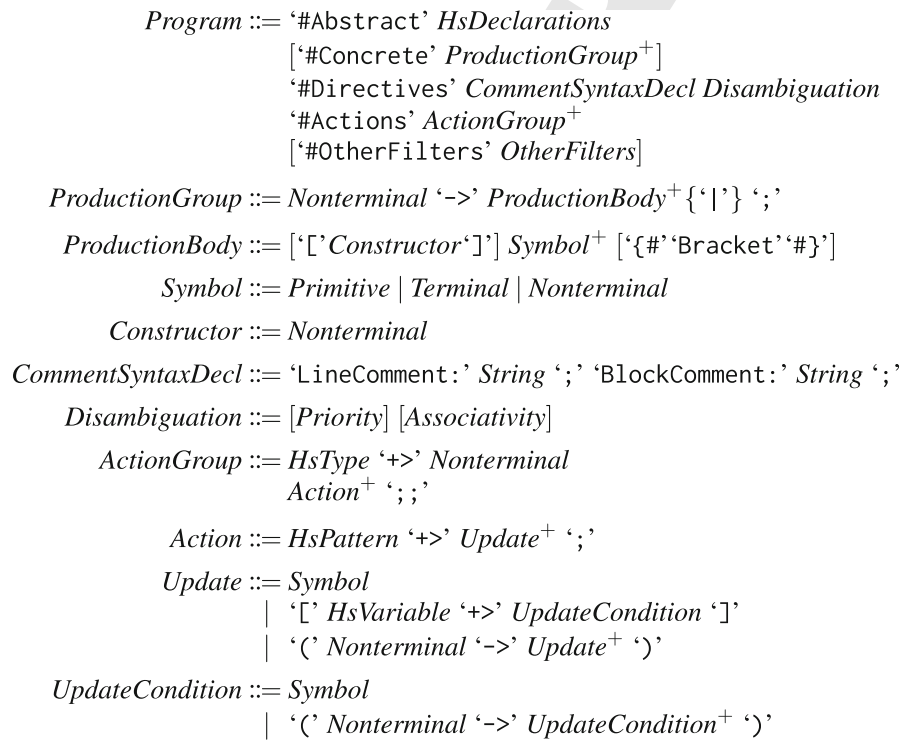

Fig. 4 Syntax of BIYACC programs. (Nonterminals with prefix Hs denote HASKell entities and follow the HASKelL syntax; the notation $n t^{+}\{$sep $\}$denotes a nonempty sequence of the same nonterminal $n t$ separated by sep. Optional elements are enclosed in a pair of square brackets. The parts relating to disambiguation and filters will be explained in Sect. 5)

Springer Ohmsha 峷親

\begin{tabular}{|l|lllll|}
\hline & Journal : Small-ext 354 & Dispatch : & $\mathbf{1 3 - 2 - 2 0 2 0}$ & Pages : 55 \\
Article No. : 82 & $\square \quad$ LE & $\square$ & TYPESET \\
& MS Code : NGCO-D-19-00023R2 & $\nabla$ & $\mathrm{CP}$ & $\square$ & DISK \\
\hline
\end{tabular}


an executable (by a HASKELL compiler) for converting between program text and

364 ASTs. Specifically:

- The abstract syntax part (decls for HASKELL data type declarations) is already valid HASKell code and is (almost) directly used as the definitions of AST data types.

- The concrete syntax part (pgs for production groups) is translated to definitions of CST data types (whose elements are representations of how a string is produced using the production rules), and also used to generate the pair of concrete parser (including a lexer) and printer for the conversion between program text and CSTs. This pair of concrete parser and printer can be shown to form an (partial) isomorphism (which will be defined in Sect. 4.1). This part will be explained in Sect. 4.2.

- The directives part (drctvs for directives) is used in the lexer for recognising single line and multi-line comments.

- The printing actions part (ags for action groups) is translated to a BIGUL program (which is a lens, see Definition 1) for handling (the semantic part of) parsing and reflective printing between CSTs and ASTs. This part will be explained in Sect. 4.3.

The whole executable is a well-behaved lens since it is the composition of an isomorphism and a lens. We will start from a recap of this fact.

\section{3}

384

\section{Composition of Isomorphisms and Lenses}

First, we give the definition of (partial) isomorphisms.

Definition 3 (Isomorphism) A (partial) isomorphism between two types $A$ and $B$ is a pair of functions:

$$
\begin{array}{r}
\text { to }:: A \rightarrow \text { Maybe } B \\
\text { from }:: B \rightarrow \text { Maybe } A
\end{array}
$$

such that the inverse properties hold:

$$
\text { to } a=\text { Just } b \quad \Leftrightarrow \quad \text { from } b=\text { Just } a .
$$

Definition 4 (Composition of isomorphism and lenses) Given an isomorphism (to and from) between $A$ and $B$ and a lens (get and put) between $B$ and $C$, we can compose them to form a new lens between $A$ and $C$, whose components get $^{\prime}$ and put' are defined by

$$
\begin{aligned}
& \text { get }^{\prime} \quad:: A \rightarrow \text { Maybe } C \\
& \text { get }^{\prime} \quad=\text { to } a \gg=\text { get } \\
& \text { put }^{\prime} \quad:: A \rightarrow C \rightarrow \text { Maybe } A \\
& \text { put }^{\prime} \text { a } c=\text { to } a \gg=\lambda b \rightarrow \text { put } b \text { } c \gg=\text { from }
\end{aligned}
$$

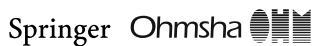

\begin{tabular}{|l|lllll|}
\hline & Journal : Small-ext 354 & Dispatch : 13-2-2020 & Pages : 55 \\
& Article No. : 82 & $\square$ LE & & $\square$ & TYPESET \\
& MS Code : NGCO-D-19-00023R2 & $\square$ CP & DISK \\
\hline
\end{tabular}


where

$$
\begin{aligned}
& (\gg=) \quad:: \text { Maybe } a \rightarrow(a \rightarrow \text { Maybe } b) \rightarrow \text { Maybe } b \\
& \text { Just } x \quad \gg=f=f x \\
& \text { Nothing } \gg=f=\text { Nothing. }
\end{aligned}
$$

This is specialised from the standard definition of lens composition [17] - an isomorphism can be lifted to a lens (with get $s=$ to $s$ and put $s v=$ from $v$ ), which can then be composed with another lens to give rise to a new lens. We thus have the following lemma.

Lemma 1 Any lens resulted from the composition in Definition 4 is well-behaved.

Therefore the whole BIYACC executable is a well-behaved lens, given that the concrete parser and printer form an isomorphism (Theorem 1) and the BIGUL program is a well-behaved lens (Theorem 2), which we will see next.

\section{The Concrete Parsing and Printing Isomorphism}

In this subsection, we describe the generation of CST data types and concrete printers (Sect. 4.2.1), the generation of concrete parsers (Sect. 4.2.2), and finally the inverse properties satisfied by the concrete parsers and printers (Sect. 4.2.3).

\section{Generating CST Data Types and Concrete Printers}

The production rules in a context-free grammar dictate how to produce strings from nonterminals, and a CST can be regarded as encoding one particular way of producing a string using the production rules. In BIYACC, we represent CSTs starting from a nonterminal $n t$ as an automatically generated HASKELL data type named $n t$, whose constructors represent the production rules for $n t$. For each of these data types, we also generate a printing function which takes a CST as input and produces a string as dictated by the production rules in the CST.

For instance, in Fig. 2, the group of production rules from the nonterminal Factor (lines 18-21) is translated to the following HASKELL data type and concrete printing function:

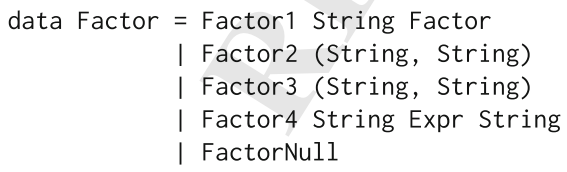


where Factor1 ... Factor4 are constructors corresponding to the four production rules, and FactorNull represents an empty CST of type Factor and is used as the default value whenever we want to create new program text depending on the view only. As an example, Factor1 represents the production rule Factor $\rightarrow$ ' '-' Factor, and its String field stores the whitespaces appearing after a negation sign in the program text. The Factor 3 case makes a call to cprtExpr:: Expr $\rightarrow$ String, which is the printing function generated for the nonterminal Expr.

Following this idea, we define the translation from production rule groups ( $p g s$ in formula (3)) to datatype definitions by source-to-source compilation rules:

$$
\begin{aligned}
& \left.\llbracket p g s \rrbracket_{\text {ProductionGroup }}=\left\langle\llbracket p g \rrbracket_{\text {ProductionGroup }}\right| p g \in \text { pgs }\right\rangle \\
& \llbracket n t \text { ' } \rightarrow \text { ' bodies } \rrbracket_{\text {ProductionGroup }}= \\
& \text { 'data' } n t \text { ' }=\text { ' }\langle\operatorname{CON}(n t, \text { body })\langle\operatorname{FIELD}(s)| s \in \text { body }\rangle \text { ' }|| \text { body } \in \text { bodies }\rangle \\
& \quad \mathrm{NULLCON}(n t) .
\end{aligned}
$$

Compilation rules of this kind will also be used later, so we introduce the notation here: compilation rules are denoted by semantic brackets $(\llbracket \cdot \rrbracket)$, and refer to some auxiliary functions, whose names are in SMALL CAPS. A nonterminal in subscript gives the 'type' of the argument or metavariable before it. The angle bracket notation $\langle f e \mid e \in e s\rangle$ denotes the generation of a list of entities of the form $f e$ for each element $e$ in the list $e s$, in the order of their appearance in es. The auxiliary function $\operatorname{con}(n t, b o d y)$ retrieves the constructor for a production rule. The fields of a constructor are generated from the right-hand side of the corresponding production rule in the way described by the auxiliary function FIELD-nonterminals that are not primitives are left unchanged (using their names for data types), primitives are stored in the String type, ${ }^{3}$ terminal symbols are dropped, and an additional String field is added for each terminal and primitive for storing layout information (whitespaces and comments) appearing after the terminal or primitive in the program text. The last step is to insert an additional empty constructor, whose name is denoted by NULLCon $(n t)$.

\section{Generating Concrete Lexers and Parsers}

The implementation of the concrete parser, which turns program text into CSTs, is further divided into two phases: lexing and parsing. In both phases, the layout information (whitespaces and comments) is automatically preserved, which makes the CSTs isomorphic to the program text.

Lexer Apart from handling the terminal symbols appearing in a grammar, the lexer automatically derived by BIYACC can also recognise several kinds of literals, including integers, strings, and identifiers, respectively, produced by the nontermi-

${ }^{3}$ The reason for storing primitives in the String type is because String is the most precise representation that will not cause the loss of any information. For instance, this is useful for retaining the leading zeros of an integer such as 073 . Storing 073 as Integer will cause the loss of the leading zero.

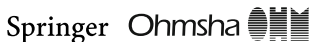

\begin{tabular}{|l|lllll|}
\hline & Journal : Small-ext 354 & Dispatch : $\mathbf{1 3 - 2 - 2 0 2 0}$ & Pages : 55 \\
Article No. : 82 & $\square$ & LE & & TYPESET \\
& MS Code : NGCO-D-19-00023R2 & $\nabla$ & CP & $\square$ & DISK \\
\hline
\end{tabular}




$$
\text { cprint }:: \mathrm{CST} \rightarrow \text { String. }
$$

496 On the other hand, from the grammar we directly use a parser generator to generate 497 a concrete parsing function

$$
\text { cparse :: String } \rightarrow \text { Maybe CST, }
$$

499

500

501

503

504

505

506

507

$$
\text { Just } \circ \text { cprint }:: \text { CST } \rightarrow \text { Maybe String. }
$$

Below we show that the inverse properties amount to the requirements that the generated parser is 'correct' and the grammar is unambiguous.

Since our concrete parsers are generated by the parser generator HAPPY [33], we need to assume that they satisfy some essential properties, for we cannot control the generation process and verify those properties.

Definition 5 (Parser correctness) A parser cparse is correct with respect to a printer cprint exactly when

$$
\text { cparse text }=\text { Just } c s t \quad \Rightarrow \quad \text { cprint cst }=\text { text }
$$

$$
\text { cprint } c s t=t e x t \quad \Rightarrow \quad \exists c s t^{\prime} . \text { cparse text }=\text { Just } c s t^{\prime} .
$$

To see what (4) means, recall that our CSTs, as described in Sect. 4.2.1, encode precisely the derivation trees, with the CST constructors representing the production rules used, and cprint traverses the CSTs and follows the encoded production rules to produce the derived program text. Now consider what cparse is supposed to do: it should take a piece of program text and find a derivation tree for it, i.e. a CST which cprints to that piece of program text. This statement is exactly (4). In other words, (4) is the functional specification of parsing, which is satisfied if the parser generator we use behaves correctly. Also it is reasonable to expect that a parser will be able to successfully parse any valid program text, and this is exactly (5).

We also need to make an assumption about concrete printers: recall that in this section we assume that the grammar is unambiguous, and this amounts to injectivity of cprint-for any piece of program text there is at most one CST that prints to it.

With these assumptions, we can now establish the isomorphism (which is rather straightforward).

Theorem 1 (Inverse Properties) If a parser cparse is correct with respect to an injective printer cprint, then cparse and Just o cprint form an isomorphism, that is,

$$
\text { cparse text }=\text { Just } c s t \quad \Leftrightarrow \quad(\text { Just } \circ \text { cprint }) \text { cst }=\text { Just } t \text { ext } \text {. }
$$

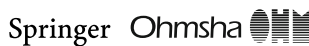

\begin{tabular}{|l|lllll|}
\hline & Journal : Small-ext 354 & Dispatch : $\mathbf{1 3 - 2 - 2 0 2 0}$ & Pages : 55 \\
& Article No. : 82 & $\square$ LE & & TYPESET \\
& MS Code : NGCO-D-19-00023R2 & $\nabla$ & CP & & DISK \\
\hline
\end{tabular}


530 Proof The left-to-right direction is immediate since the right-hand side is 531 equivalent to cprint $c s t=t e x t$, and the whole implication is precisely (4). For the 532 right-to-left direction, again the antecedent is equivalent to cprint cst $=$ text, and we 533 can invoke (5) to obtain cparse text $=$ Just $c s t^{\prime}$ for some $c s t^{\prime}$. This is already close 534 to our goal-what remains to be shown is that $c s t^{\prime}$ is exactly $c s t$, which is indeed the 535 case because

$$
\begin{aligned}
& \text { cparse text }=\text { Just } c s t^{\prime} \\
\Rightarrow \quad & \{\text { antecedent }\} \\
& \text { cparse }(\text { cprint cst })=\text { Just } c s t^{\prime} \\
\Rightarrow \quad & \{(4)\} \\
& \text { cprint cst } t^{\prime}=\text { cprint } c s t \\
\Rightarrow \quad & \{\text { cprint is injective }\} \\
& \text { cst } t^{\prime}=\text { cst } .
\end{aligned}
$$

\section{Generating the BIGUL Lens}

The source-to-source compilation from the actions part of a $\mathrm{BIYACC}$ program to a BiGUL program (i.e. lens) is shown in Fig. 5. Additional arguments to the semantic bracket are typeset in superscript, and the notation $\langle\ldots \mid \ldots \in \ldots\rangle\{s\}$ means inserting $s$ between the elements of the list.

Action groups Each group of actions is translated into a small BiGUL program, whose name is determined by the view type $v t$ and source type $s t$ and denoted by $\operatorname{PROG}(v t, s t)$. The BIGUL program has one single Case statement, and each action is translated into two branches in this Case statement, one normal and the other adaptive. All the adaptive branches are gathered in the second half of the Case statement, so that the normal branches will be tried first. For example, the third group of type Arith $+>$ Factor is compiled to

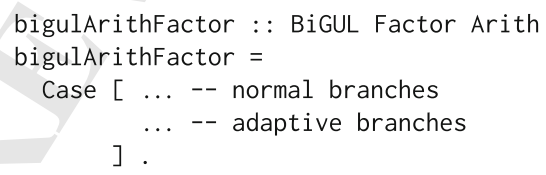

552 Normal branches We said in Sect. 2 that the semantics of an action is to perform 553 pattern matching on both the source and view, and then update parts of the source 554 with parts of the view. This semantics is implemented with a normal branch: the 555 source and view patterns are compiled to the main condition, and, together with the 556 updates overlaid on the source pattern, also to an update operation. For example, the 557 first action in the Arith-Factor group

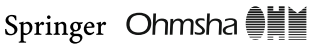

\begin{tabular}{lllll} 
Journal : Small-ext 354 & Dispatch : & $\mathbf{1 3 - 2 - 2 0 2 0}$ & \multicolumn{2}{l}{ Pages : $\mathbf{5 5}$} \\
Article No. $: \quad \mathbf{8 2}$ & $\square \quad$ LE & & $\square$ & TYPESET \\
MS Code : NGCO-D-19-00023R2 & $\square$ & CP & $\square$ & DISK
\end{tabular}




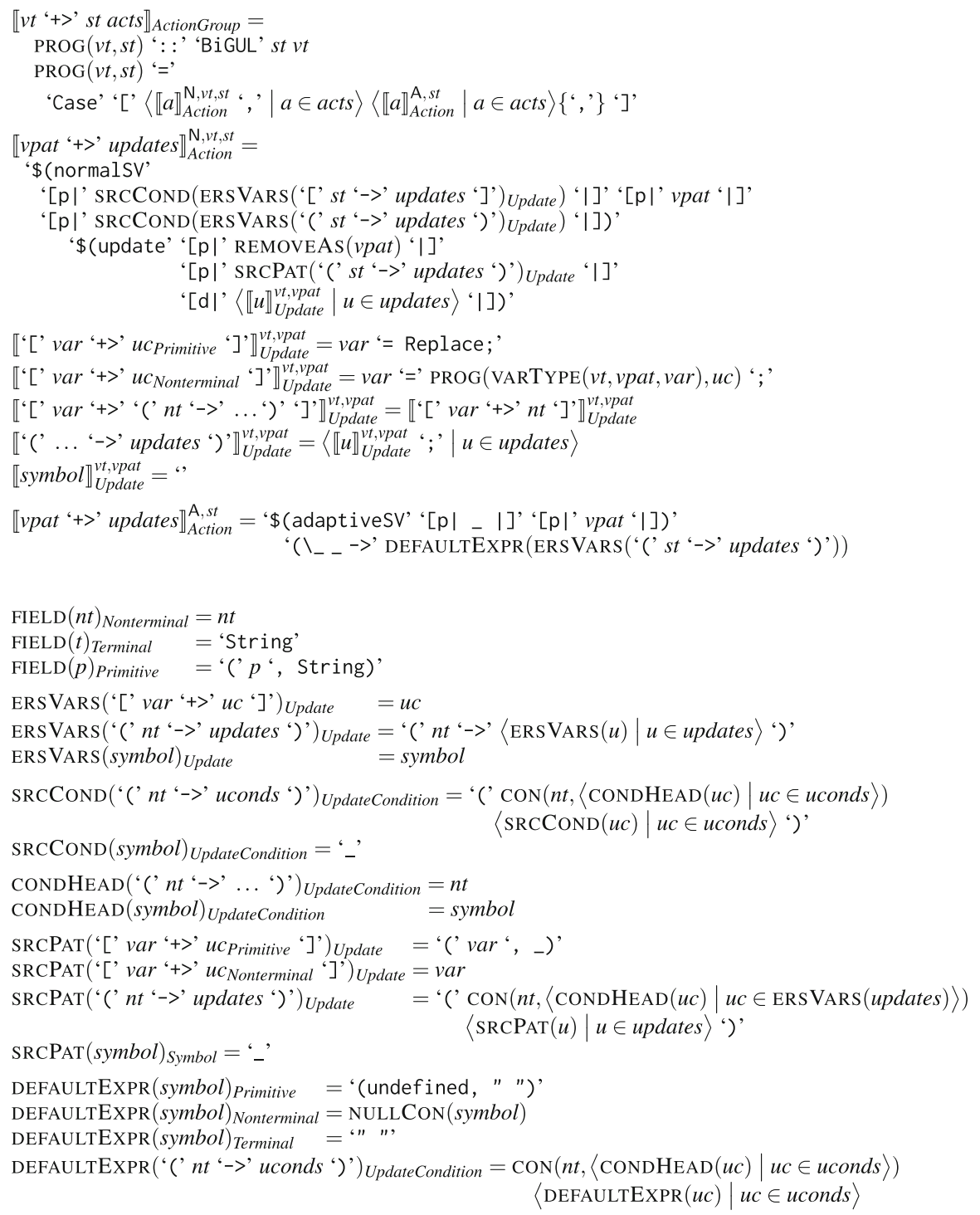

Fig. 5 Semantics of BIYACC programs (as BiGUL programs)

$$
\operatorname{Sub}(\text { Num } 0) \text { y }+>\text { '-' (y }+>\text { Factor })
$$

559 is compiled to

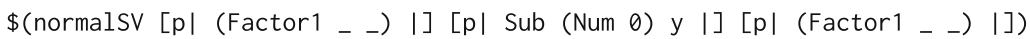

$\$$ (update $[\mathrm{p} \mid$ Sub (Num 0) y |] [p| (Factor1 - y) |] [d| y = bigulArithFactor; |]).

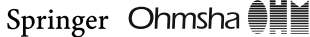

\begin{tabular}{|l|llll|}
\hline & Journal : Small-ext 354 & Dispatch : & $\mathbf{1 3 - 2 - 2 0 2 0}$ & Pages : 55 \\
Article No. : 82 & $\square \quad$ LE & $\square$ & TYPESET \\
& MS Code : NGCO-D-19-00023R2 & $\square \quad C P$ & $\square$ & DISK \\
\hline
\end{tabular}


561

562

563

564

565

566

567

568

569

570

571

573

574

575

576

577

578

579

580

581

582

583

584

585

587

588

589

590

591

592

593

\section{4}

When the CST is a Factor1 and the AST matches Sub (Num 0) y, we enter this branch, decompose the source and view by pattern matching, and use the view's right subtree y to update the second field of the source while skipping the first field (which stores whitespaces); the name of the BIGUL program for performing the update is determined by the type of the smaller source y (deduced by VARTYPE) and that of the smaller view.

Adaptive branches When all actions in a group fail to match, we should adapt the source into a proper shape to correspond to the view. This is done by generating adaptive branches from the actions during compilation. For example, besides a normal branch, the first action in the Arith-Factor group $\operatorname{Sub}($ Num 0$) y+>$ ' - ' $(y+>$ Factor $)$ is also compiled to

$\$($ adaptiveSV $[p|\ldots|][p \mid \operatorname{Sub}($ Num 0$) \ldots \mid])\left({ }_{-} \ldots\right.$ - Factor1 " FactorNull) .

Since the source pattern of the main condition (of the adaptive branch) is a wildcard, the branch is always applicable if the view matches Sub (Num 0)_. The body of the adaptation function is generated by the auxiliary function DEFAULTEXPR, which creates a skeletal value-here Factor1 " FactorNull represents a negation skeleton whose value is not (recursively) created yet-that matches the source pattern. These adaptive branches are placed at the end of an action group and tried only if no normal branches are applicable so that unnecessary adaptation will never be performed.

Entry point The entry point of the program is chosen to be the BiGUL program compiled from the first group of actions. This corresponds to our assumption that the initial input concrete and abstract syntax trees are of the types specified for the first action group. (It is rather simple so the rules are not shown in the figure.) For the expression example, we generate a definition

\section{entrance $=$ bigulArithExpr}

which is invoked in the main program.

Well-behavedness Since BiGUL programs always denote well-behaved lenses, a fact which has been formally verified [39], we get the following theorem for free.

Theorem 2 (Well-behavedness) The BIGUL program generated from a BIYACC program is a lens, that is, it satisfies the well-behavedness laws in Definition 1 with cst substituted for the source s and ast for the view $v$ :

$$
\begin{array}{rll}
\text { put cst ast }=\text { Just } c s t^{\prime} & \Rightarrow & \text { get cst } t^{\prime}=\text { Just } a s t \\
\text { get cst }=\text { Just } a s t & \Rightarrow & \text { put cst ast }=\text { Just } c s t .
\end{array}
$$

\section{Handling Grammatical Ambiguity}

In Sect. 4, we have described the basic version of BIYACC, about which there is an important assumption (stated in Theorem 1) that grammars have to be unambiguous. Having this assumption can be rather inconvenient in practice, however, as

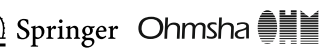

$\begin{array}{llll}\text { Journal : Small-ext 354 } & \text { Dispatch : 13-2-2020 } & \text { Pages : 55 } \\ \text { Article No. : } \mathbf{8 2} & \square \text { LE } & \square \text { TYPESET }\end{array}$




\begin{tabular}{|c|c|c|}
\hline \multicolumn{3}{|c|}{ \#Concrete } \\
\hline & | [Minus] & Expr '-' Expr \\
\hline & | [Times] & Expr '*' Expr \\
\hline & | [Division] & Expr '/' Expr \\
\hline & | [Paren] & '('Expr ')' \\
\hline & | [Lit] & Numeric \\
\hline
\end{tabular}

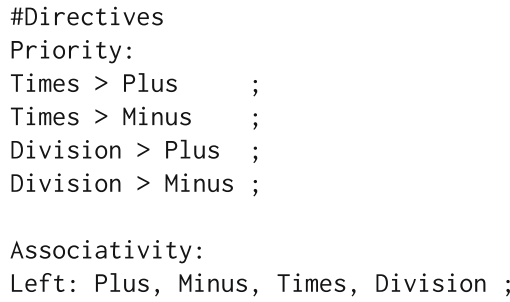

\#Directives

Priority:

Times > Plus

Division $>$ Plus

Associativity

Left: Plus, Minus, Times, Division

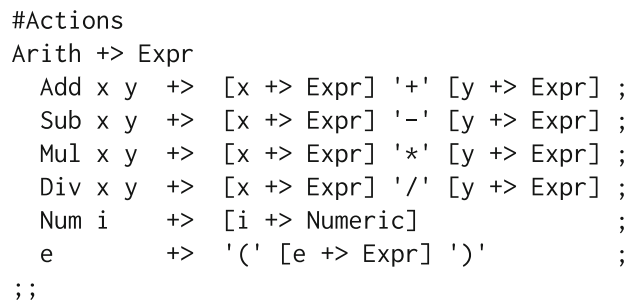

Fig. 6 Arithmetic expressions defined by an ambiguous grammar and the corresponding printing actions. (For simplicity, the variable and negation productions are omitted)

ambiguous grammars (with disambiguation directives) are often preferred since they are considered more natural and human friendly than their unambiguous versions $[2,26]$. Therefore, the purpose of this section is to revise the architecture of basic BIYACC to allow the use of ambiguous grammars and disambiguation directives. This is in fact a long-standing problem: tools designed for building parser and printer pairs usually do not support such functionality (Sect. 7.1).

For example, consider the ambiguous grammar (with disambiguation directives) and printing actions in Fig. 6, which we will refer to throughout this section. Note that the parenthesis structure is dropped when converting a CST to its AST (as stated by the last printing action of Arith+> Expr). The grammar is converted to CST data types and constructors as in Sect. 4.2.1, but here we explicitly give names such as Plus and Times to production rules, and these names (instead of automatically generated ones) are used for constructors in CSTs. Compared with this grammar, the unambiguous one shown in Fig. 2 is less intuitive as it uses different nonterminals to resolve the ambiguity regarding operator precedence and associativity.

In this section, we explain the problem brought by ambiguous grammars (Sect. 5.1) and address it (Sect. 5.2) using generalised parsing and bidirectionalised filters (bifilters for short). Then we extend BIYACC with bi-filters (Sect. 5.3) while still retaining the well-behavedness. To program with bi-filters easily, we provide compositional bi-filter directives (Sect. 5.4) which compile to priority and associativity bi-filters. Power users can also define their own bi-filters (Sect. 5.5), and we illustrate this by writing a bi-filter that solves the (in)famous dangling-else problem.

\section{Problems with Ambiguous Grammars}

Consider the original architecture of BIYACC in Fig. 3, which we want to (and basically will) retain while adapting it to support ambiguous grammars. The first

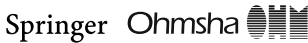

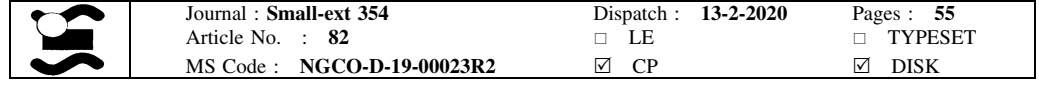


component (of the executable) we should adapt is cparse $::$ String $\rightarrow$ Maybe CST, the (concrete) parsing direction of the isomorphism: since there can be multiple CSTs corresponding to the same program text, cparse needs to choose one of them as the result. Disambiguation directives [23] were invented to describe how to make this choice. For example, with respect to the grammar in Fig. 6, text $1+2{ }^{*} 3$ will have either of the two CSTs ${ }^{4}$ :

$$
\begin{aligned}
& \text { cst }_{1}=\sharp \text { Plus } 1 \text { (Times } 23 \text { ) } \\
& \text { cst } \left._{2}=\sharp \text { Times (Plus } 112\right) 3
\end{aligned}
$$

depending on the precedence of addition and multiplication. Conventionally, we can use the YACC-style disambiguation directives \%left '+'; \%left '*'; to specify that multiplication has higher precedence over addition, and instruct the parser to choose cst $_{1}$.

However, merely adapting cparse with disambiguation behaviour is not enough, since the isomorphism (Theorem 1), in particular its right to left direction (which is simplified as cparse ( cprint cst) $=$ Just $c s t$ ) cannot be established when an ambiguous grammar is used-in the example above, cparse (cprint cst ${ }_{2}$ ) = Just cst $_{1} \neq$ Just cst $_{2}$. This is because the image of cparse is strictly smaller than the domain of cprint: if we start from any CST not in the image of cparse, we will never be able to get back to the same CST through cprint and then cparse. This tells us that, to retain the isomorphism, the domain of cprint should not be the whole CST but only the image of cparse, i.e. the set of valid CSTs (as defined by the disambiguation directives), which we denote by $\mathrm{CST}_{F}$ (for reasons that will be made clear in Sect. 5.3).

Now that the right-hand side domain of the isomorphism is restricted to $\operatorname{CsT}_{F}$, the source of the lens should be restricted to this set as well. For get $::$ CST $\rightarrow$ Maybe AST we need to restrict its domain, which is easy; for put $::$ CST $\rightarrow$ AST $\rightarrow$

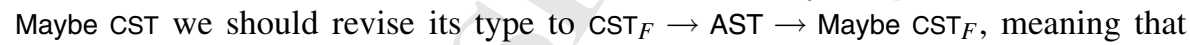
put should now guarantee that the CSTs it produces are valid, which is nontrivial. For example, consider the result of put cst ast where ast $=$ Mul (Add (Num 1) (Num 2)) (Num 3) and cst is some arbitrary tree. A natural choice is cst $_{2}$, which, however, is excluded from $\operatorname{CST}_{F}$ by disambiguation. A possible solution could be making put refuse to produce a result from ast, but this is unsatisfactory since ast is perfectly valid and should not be ignored by put. A more satisfactory way is creating a CST with proper parentheses, like cst ${ }_{3}={ }^{\sharp}$ Times (Paren (Plus 12$)$ ) 3 . But it is not clear in what cases parentheses need to be added, in what cases they need not, and in what cases they cannot.

We are now led to a fundamental problem: generally, put strategies for producing valid CSTs should be inferred from the disambiguation directives, but the semantics of YACC disambiguation directives are defined over the implementation of YACC's underlying LR parsing algorithm with a stack [3,23], and therefore it is nontrivial to invent a dual semantics in the put direction. To have a simple and clear semantics of

\footnotetext{
${ }^{4}$ For simplicity, we use ${ }^{\sharp}$ to annotate type-incorrect CSTs in which fields for layouts (and comments) and unimportant constructors such as Lit are omitted.
}

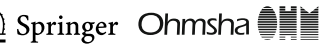

\begin{tabular}{lllll} 
Journal : Small-ext 354 & Dispatch : & $\mathbf{1 3 - 2 - 2 0 2 0}$ & \multicolumn{2}{c}{ Pages : $\mathbf{5 5}$} \\
Article No. $: \mathbf{8 2}$ & $\square$ LE & & $\square$ & TYPESET \\
MS Code : NGCO-D-19-00023R2 & $\square$ & CP & $\square$ DISK \\
\hline
\end{tabular}


the disambiguation process, we turn away from YACC's traditional approach and opt for an alternative approach based on generalised parsing with disambiguation filters $[9,26]$, whose semantics can be specified implementation independently. Based on this simple and clear semantics, we will be able to devise ways to amend put to produce only valid CSTs, and formally state the conditions under which the executable generated by the revised BIYACC is well behaved.

\section{Generalised Parsing and Bidirectionalised Filters}

The idea of generalised parsing is for a parser to produce all possible CSTs corresponding to its input program text instead of choosing only one CST (possibly prematurely) [14, 47, 50, 54], and works naturally with ambiguous grammars. In practice, a generalised parser can be generated using, e.g., HAPPY's GLR mode [33], and we will assume that given a grammar we can obtain a generalised parser:

$$
\text { cgparse }:: \text { String } \rightarrow[\mathrm{CST}] .
$$

The result of cgparse is a list of CSTs. We do not need to wrap the result type in Maybe-if cgparse fails, an empty list is returned. And we should note that, while the result is a list, what we really mean is a set (commonly represented as a list in HASKELL) since we do not care about the order of the output CSTs and do not allow duplicates.

With generalised parsing, program text is first parsed to all the possible CSTs; disambiguation then becomes an extremely simple concept: removing CSTs that the user does not want. One possible semantics of disambiguation may be a function judge $::$ Tree $\rightarrow$ Bool; during disambiguation, this function is applied to all candidate CSTs, and a candidate cst is removed if judge cst returns False, or kept otherwise. We call these functions disambiguation filters ('filters' for short). ${ }^{5}$ For example, to state that top-level addition is left-associative, we can use the following filter ${ }^{6}$ to reject right-sided trees:

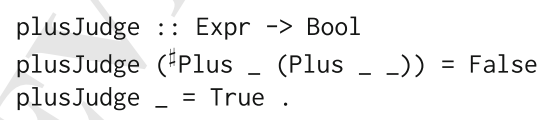

This simple and clean semantics of disambiguation is then amenable to 'bidirectionalisation', which we do next.

Note that, unlike YACC's disambiguation directives, which assign precedence and associativity to individual tokens and implicitly exclude 'some' CSTs, in plusJudge above we explicitly ban incorrect CSTs through pattern matching. Having described which CSTs are incorrect, we can further specify what to do with incorrect CSTs in

\footnotetext{
5 The general type for disambiguation filters is $[t] \rightarrow[t]$, which allows comparison among a list of CSTs. However, since in this paper we only consider property filters defined in terms of predicates (on a single tree), it is sufficient to use the simplified type $t \rightarrow$ Bool. See Sect. 7.2.

${ }^{6}$ This is not a very realistic filter, although it sufficiently demonstrates the use of filters and removes ambiguity in simplest cases like $1+2 * 3$. In general, the filter should be complete (Definition 9 ) so that ambiguity is fully removed from the grammar.
}

\section{Springer Ohmsha 譬咅}

\begin{tabular}{|l|lllll|}
\hline & Journal : Small-ext 354 & Dispatch : $\mathbf{1 3 - 2 - 2 0 2 0}$ & Pages : 55 \\
& Article No. : 82 & $\square$ & LE & & TYPESET \\
& MS Code : NGCO-D-19-00023R2 & $\nabla$ & CP & $\square$ & DISK \\
\hline
\end{tabular}


697 the printing direction. Whenever a CST 'in a bad shape', i.e. rejected by a filter like

698 plusJudge, is produced, we can repair it so that it becomes 'in a good shape':

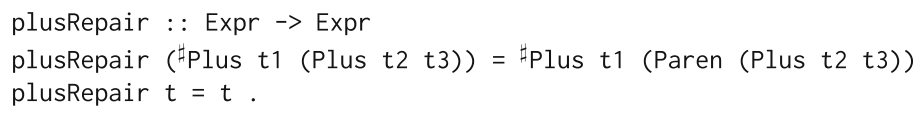

700 The above function states that whenever a Plus is another Plus's right child, there 701 must be a parenthesis structure Paren in between. Observant readers might have 702 found that the trees processed by plusJudge and plusRepair have the same pattern. 703 We can therefore pair the two functions and make a bidirectionalised filter ('bi704 filters' for short):

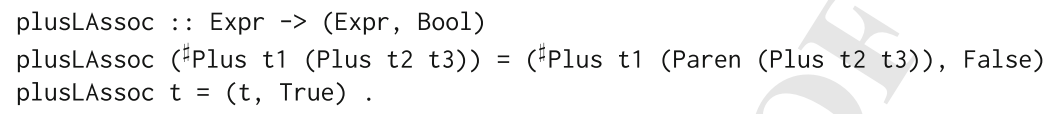

706 But there is still some redundancy in the definition of plusLAssoc, for when the 707 input tree is correct we always return the same input tree; this can be further 708 optimised:

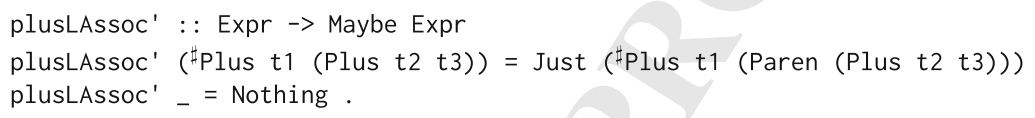

710 Generalising the example above, we arrive at the definition of bi-filters.

711 Definition 6 (Bidirectionalised filters) A bidirectionalised filter $F$ working on trees 712 of type $t$ is a function of type BiFiltert defined by:

$$
\text { type BiFilter } t=t \rightarrow \text { Maybe } t
$$

714 satisfying

$$
\text { repair } F t=t^{\prime} \quad \Rightarrow \quad \text { judge } F t^{\prime}=\text { True }
$$

716 where the two directions repair and judge are defined by:

$$
\begin{array}{r}
\text { repair }:: \text { BiFilter } t \rightarrow(t \rightarrow t) \\
\text { repair } F t=\text { case } F t \text { of } \\
\text { Nothing } \rightarrow t \\
\text { Just } t^{\prime} \rightarrow t^{\prime} \\
\text { judge }:: \text { BiFilter } t \rightarrow(t \rightarrow \text { Bool }) \\
\text { judge } F t=\text { case } F t \text { of } \\
\text { Nothing } \rightarrow \text { True } \\
\text { Just_ } \rightarrow \text { False . }
\end{array}
$$

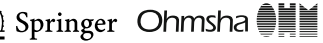

\begin{tabular}{lllll} 
Journal : Small-ext 354 & Dispatch : & $\mathbf{1 3 - 2 - 2 0 2 0}$ & \multicolumn{2}{l}{ Pages : 55 } \\
Article No. $: \mathbf{8 2}$ & $\square$ LE & & $\square$ & TYPESET \\
MS Code : NGCO-D-19-00023R2 & $\square$ & CP & $\square$ & DISK \\
\hline
\end{tabular}


The functions repair and judge accept a bi-filter and return, respectively, the specialised repair and judge functions for that bi-filter. For clarity, we let repair ${ }_{F}$ denote repair $F$ and let judge $F$ denote judge $F$. The bi-filter law RepairJudge dictates that repair $_{F}$ should transform its input tree into a state accepted by judge $e_{F}$. The reader may wonder why there is not a dual JudgeRepair law saying that if a tree is already of an allowed form justified by judge $e_{F}$, then repair $_{F}$ should leave it unchanged. In fact, this is always satisfied according to the definitions of judge and repair, so we formulate it as a lemma.

Lemma 2 (JudgeRepair) Any bi-filter F satisfies the JudgeRepair property:

$$
\text { judge }_{F} t=\text { True } \Rightarrow \text { repair }_{F} t=t .
$$

Proof From judge $_{F} t=$ True we deduce $F t=$ Nothing, which implies repair $F=t$.

In the next section, we will describe how to fit generalised parsers and bi-filters into the architecture of BIYACC. To let bi-filters work with the lens between CSTs and ASTs, we require a further property characterising the interaction between the repairing direction of a bi-filter and the get direction of a lens.

Definition 7 (PassThrough) A bi-filter $F$ satisfies the PassThrough property with respect to a function get exactly when

$$
\text { get } \circ \text { repair }_{F}=\text { get. }
$$

If we think of a get function as mapping CSTs to their semantics (in our case ASTs), then the PassThrough property is a reasonable requirement since it guarantees that the repaired CST will have the same semantics as before (since it is converted to the same AST). This property will be essential for establishing the well-behavedness of the executable generated by the revised BIYACC.

\section{The New BIYACC System for Ambiguous Grammars}

As depicted in Fig. 7, the executable generated by the new BIYACC system is still the composition of an isomorphism and a lens, which is the structure we have tried to retain. To precisely identify the changes in several generated components (in the executable file) and demonstrate how parsing and printing work with a bi-filter, we present Fig. 8 and will use this one instead. In the new system, we will still use the get and put transformations generated from printing actions and the concrete printer cprint from grammars, while the concrete parser cparse is replaced with a generalised parser cgparse. Additionally, the \#Directives and \#OtherFilters parts will be used to generate a bi-filter $F$, whose $j u d g e_{F}$ (used in the selectBy $y_{F}$ function in Fig. 8) and repair $_{F}$ components are integrated into the isomorphism and lens parts

\begin{tabular}{|l|lllll|}
\hline & Journal : Small-ext 354 & Dispatch : $\mathbf{1 3 - 2 - 2 0 2 0}$ & Pages : 55 \\
& Article No. : 82 & $\square$ LE & & $\square$ & TYPESET \\
& MS Code : NGCO-D-19-00023R2 & $\nabla$ & CP & & DISK \\
\hline
\end{tabular}




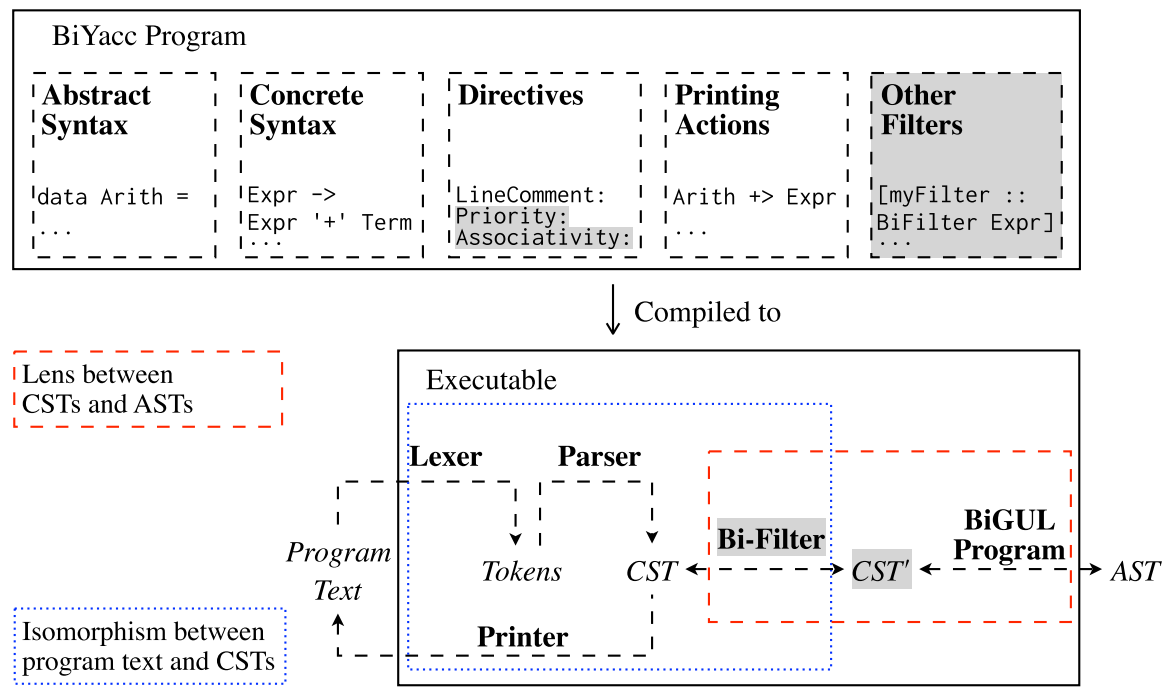

Fig. 7 New architecture of BIYACC (new components are in light grey)

Isomorphism between text and cst

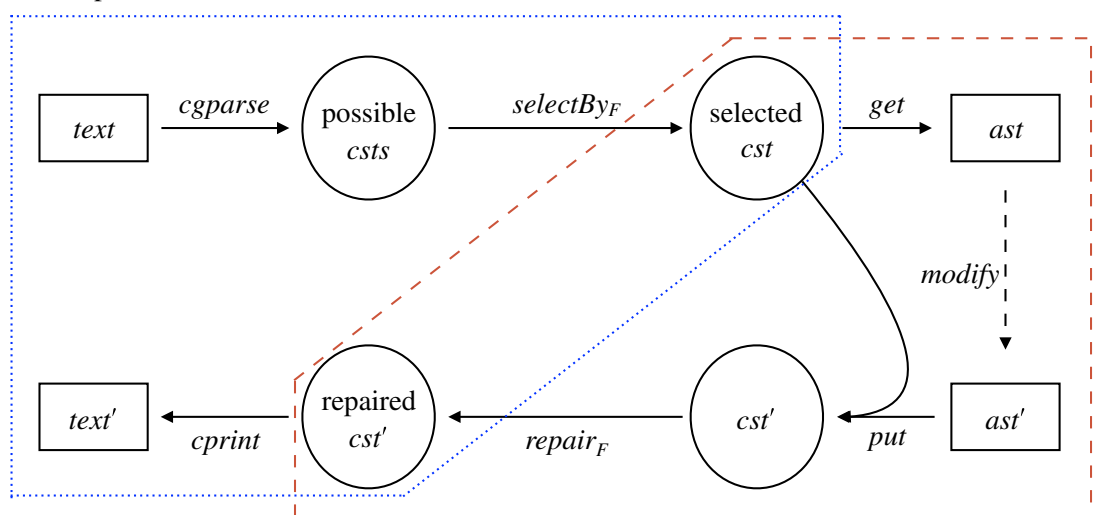

Fig. 8 A schematic diagram showing how parsing and printing work with a bi-filter

753 respectively, so that the right-hand side domain of the isomorphism and the source 754 of the lens become CST $_{F}$, the set of valid CSTs:

$$
\mathrm{CST}_{F}=\left\{c s t \in \mathrm{CST} \mid \text { judge }_{F} \text { cst }=\text { True }\right\} .
$$

756 Next, we introduce the (new) isomorphism and lens parts, and prove their inverse 757 properties and well-behavedness, respectively.

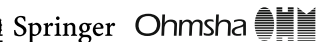

\begin{tabular}{|l|lllll|}
\hline & Journal : Small-ext 354 & Dispatch : 13-2-2020 & Pages : $\mathbf{5 5}$ \\
Article No. : 82 & $\square$ LE & $\square$ & TYPESET \\
& MS Code : NGCO-D-19-00023R2 & $\nabla$ CP & $\square$ & DISK \\
\hline
\end{tabular}


763

764

765

766

767

768

769

770

772

\section{The Revised Isomorphism between Program Text and CSTs}

Let us first consider the isomorphism part between String and $\mathrm{CST}_{F}$, which is enclosed within the blue dotted lines in Fig. 8 and consists of cprint, cgparse, and selectBy $y_{F}$ :

$$
\begin{aligned}
& \text { cprint }:: \mathrm{CST} \rightarrow \text { String } \\
& \text { cgparse }:: \text { String } \rightarrow[\mathrm{CST}]
\end{aligned}
$$

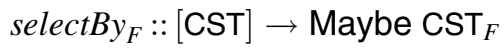

$$
\begin{aligned}
& \text { selectBy }_{F} \text { csts }=\text { case } \text { selectBy judge }_{F} \text { csts of } \\
& {[c s t] \rightarrow \text { Just } c s t} \\
& \rightarrow \text { Nothing } \\
& \text { selectBy }::(a \rightarrow \mathrm{Bool}) \rightarrow[a] \rightarrow[a] \\
& \text { selectBy } p[]=[] \\
& \text { selectBy } p(x: x s) \mid p x=x: \text { selectBy } p \text { xs } \\
& \text { selectBy } p(x: x s) \mid \text { otherwise }=\text { selectBy } p \text { xs . }
\end{aligned}
$$

In the parsing direction, first cgparse produces all the CSTs; then selectBy $y_{F}$ utilises a function selectBy and a predicate judge $e_{F}$ to (try to) select the only correct $c s t$; if there is no correct CST or more than one correct CST, Nothing is returned. The function selectBy, which selects from the input list exactly the elements satisfying the given predicate, is named filter in HASKELL's standard libraries but renamed here to avoid confusion. In the printing direction, we still use cprint to flatten a (correct) CST back to program text. Formally, constructed from cgparse and cprint, the two directions of the isomorphism are:

$$
\begin{array}{lll}
\text { pparse }_{F} & :: & \text { String } \rightarrow \text { Maybe CST } \\
\text { pparse }_{F} & = & \text { selectBy }_{F} \circ \text { cgparse } \\
\text { print }_{F} & :: & \text { CST }_{F} \rightarrow \text { Maybe String } \\
\text { cprint }_{F}= & \text { Just } \circ \text { cprint } .
\end{array}
$$

We are eager to give the revised version of the inverse properties (Theorem 3) and their proofs, which, however, depend on two assumptions about generalised parsers and bi-filters. So let us present them in order.

Definition 8 (Generalised parser correctness) A generalised parser cgparse is correct with respect to a printer cprint exactly when

$$
\text { cgparse text }=\{\text { cst } \in \mathrm{CST} \mid \text { cprint cst }=\text { text }\} .
$$

This is exactly Definition 3.7 of Klint and Visser [26]. We remind the reader again that we use sets and lists interchangeably for the parsing results.

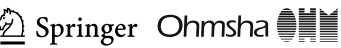

\begin{tabular}{|l|lllll|}
\hline & Journal : Small-ext 354 & Dispatch : $\mathbf{1 3 - 2 - 2 0 2 0}$ & Pages : 55 \\
& Article No. : 82 & $\square$ & LE & & TYPESET \\
& MS Code : NGCO-D-19-00023R2 & $\nabla$ & CP & $\square$ & DISK \\
\hline
\end{tabular}


780 Definition 9 (Bi-filter completeness) A bi-filter $F$ is complete with respect to a

781 printer cprint exactly when

$$
\text { text } \in \text { Img cprint } \Rightarrow \mid\left\{\text { cst } \in \mathrm{CST}_{F} \mid \text { cprint } \operatorname{cst}=\text { text }\right\} \mid=1 .
$$

$783(\operatorname{Img} f=\{y \mid \exists x . f x=y\}$ is the image of the function $f$.)

784 This is revised from Definition 4.3 of Klint and Visser [26], where they require 785 that filters select exactly one CST and reject all the others. Since it is undecidable to 786 judge whether a given context-free grammar is ambiguous [10], we cannot tell 787 whether a (bi-)filter (for the full CFG) is complete, either. But still, some checks can 788 be performed in simple cases, as stated in Sect. 7.

789 The following two lemmas connect our two assumptions, Definitions 8 and 9, 790 with the definitions of cparse $_{F}$ and cprint $_{F}$.

791 Lemma 3 Given cparse $_{F}$ and cprint $_{F}$ where cgparse is correct and $F$ is complete 792 with respect to cprint, we have text $\in$ Img cprint $\Rightarrow \quad \exists$ cst $\in \mathrm{CST}_{F}$. cparse $_{F}$ text $=$ Just $c s t \wedge$ cprint $c s t=$ text.

\section{Proof We reason:}

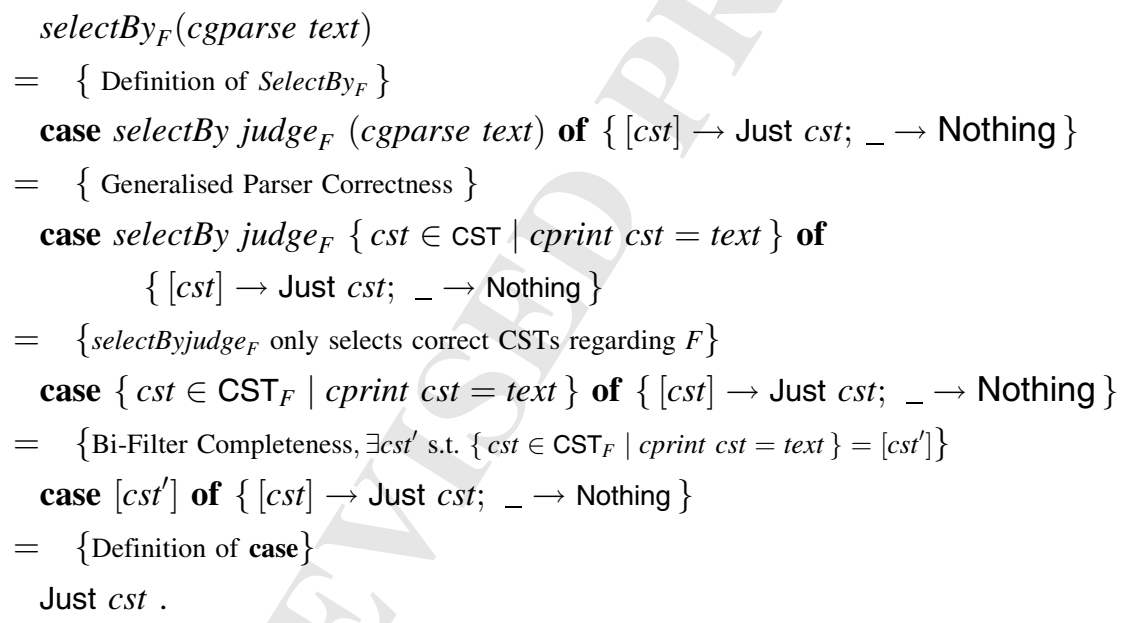

797 Moreover, cst satisfies cprint cst $=$ text, since the latter is the comprehension con798 dition of the set from which $c s t$ is chosen, and therefore cprint $_{F} c s t=$ Just text.

799 Lemma 4 (Printer injectivity) If $F$ is a complete bi-filter, then cprint $_{F}$ is injective. $^{2}$

800 Proof Assume that $c s t, c s t^{\prime} \in \mathrm{CsT}_{F}$ and cprint $c s t=$ cprint $\mathrm{cst}^{\prime}=$ text for some 801 text; that is, both cst and $c s t^{\prime}$ are in the set $P=\left\{c s t \in \mathrm{CST}_{F} \mid\right.$ cprint cst $=$ text $\}$. 802 Since text $\in$ Img cprint, by the completeness of $F$ we have $|P|=1$, and hence $803 c s t=c s t^{\prime}$. 
We can now prove a generalised version of Theorem 1 for ambiguous grammars.

Theorem 3 (Inverse properties with bi-Filters) Given cparse ${ }_{F}$ and cprint $_{F}$ where cgparse is correct and $F$ is complete, we have the following:

$$
\begin{aligned}
& \text { cparse }_{F} \text { text }=\text { Just cst } \Rightarrow \text { cprint }_{F} \text { cst }=\text { Just text } \\
& \text { cprint }_{F} c s t=\text { Just text } \Rightarrow \text { cparse }_{F} \text { text }=\text { Just } c s t .
\end{aligned}
$$

Proof For (6): let Just $c s t=$ selectBy $_{F}$ (cgparse text). According to the definition of selectBy $_{F}$, we have cst $\in$ cgparse text. By Generalised Parser Correctness cprint $c s t=$ text, and therefore cprint $_{F}$ cst $=$ Just text.

For (7): the antecedent implies cprint cst $=$ text. By Lemma 3, we have cparse $_{F}$ text $=$ Just $c s t^{\prime}$ for some $c s t^{\prime} \in \operatorname{csT}_{F}$ such that cprint $_{F} c s t^{\prime}=$ Just text $=$ cprint $_{F} c s t$. By Lemma 4 we know $c s t^{\prime}=c s t$, and thus cparse $_{F}$ text $=$ Just $c s t$.

\section{The Revised Lens between CSTs and ASTs}

Recall that the \#Action part of a BIYACC program produces a lens (BIGUL program) consisting of a pair of well-behaved get and put functions:

$$
\begin{aligned}
& \text { get }:: \text { CST } \rightarrow \text { Maybe AST } \\
& \text { put }:: \text { CST } \rightarrow \text { AST } \rightarrow \text { Maybe CST . }
\end{aligned}
$$

To work with a bi-filter $F$, in particular its repair $_{F}$ component, they need to be adapted to $\operatorname{get}_{F}$ and $\mathrm{put}_{F}$, which accept only valid CSTs:

$$
\begin{array}{ll}
\text { get }_{F} & :: \text { CST }_{F} \rightarrow \text { Maybe AST } \\
\text { get }_{F} & =\text { get } \\
\text { put }_{F} & :: \text { CST }_{F} \rightarrow \text { AST } \rightarrow \text { Maybe CST }_{F} \\
\text { put }_{F} \text { cst ast } & =\text { fmap repair }_{F} \text { (put cst ast) }
\end{array}
$$

where fmap is a standard HASKELL library function defined (for Maybe) by

$$
\begin{array}{ll}
\text { fmap } & :: \quad(a \rightarrow b) \rightarrow \text { Maybe } a \rightarrow \text { Maybe } b \\
\text { fmapf Nothing } & =\text { Nothing } \\
\text { fmapf (Justx) } & =\operatorname{Just}(f x) .
\end{array}
$$

We will need a lemma about fmap, which can be straightforwardly proved by a case analysis.

Lemma 5 If fmap $f m x=$ Just $y$, then there exists $x$ such that $m x=$ Just $x$ and $f x=y$.

Now we prove that get $_{F}$ and $p u t_{F}$ are well-behaved, which is a generalisation of Theorem 2 for ambiguous grammars.

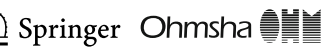

\begin{tabular}{|l|lllll|}
\hline & Journal : Small-ext 354 & Dispatch : 13-2-2020 & Pages : 55 \\
Article No. : 82 & $\square$ LE & $\square$ & TYPESET \\
& MS Code : NGCO-D-19-00023R2 & $\square$ CP & $\square$ & DISK \\
\hline
\end{tabular}


832 Theorem 4 (Well-behavedness with bi-filters) Given a complete bi-filter $F$ and a 833 well-behaved lens consisting of get and put, if get and $F$ additionally satisfy

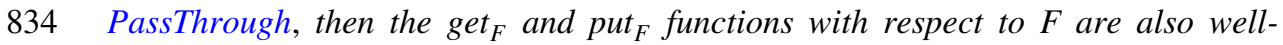
835 behaved:

$$
\begin{aligned}
& \text { put }_{F} \text { cst ast }=\text { Just } c s t^{\prime} \Rightarrow \text { get }_{F} c s t^{\prime}=\text { Just } a s t \\
& \text { get }_{F} c s t=\text { Just } a s t \quad \Rightarrow \quad \text { put }_{F} \text { cstast }=\text { Just } c s t .
\end{aligned}
$$

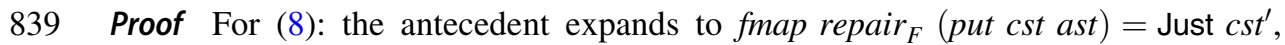
840 which, by Lemma 5, implies put cst ast = Just $c s t^{\prime \prime}$ for some $c s t^{\prime \prime}$ such that 841 repair $_{F} c s t^{\prime \prime}=c s t^{\prime}$. Now we reason:

$$
\begin{aligned}
& \text { get }_{F} \text { cst }^{\prime} \\
& =\left\{\text { Definition of } \text { get }_{F} \text { and } c s t \in \mathrm{CsT}_{F}\right\} \\
& \text { get } \text { cst }^{\prime} \\
& =\left\{\text { Definition of } c s t^{\prime}\right\} \\
& \text { get }\left(\text { repair }_{F} \text { cst }^{\prime \prime}\right) \\
& =\{\text { PassThrough }\} \\
& \text { get cst }{ }^{\prime \prime} \\
& =\{\text { PutGet }\} \\
& \text { Just } \text { ast . }
\end{aligned}
$$

843 For (9):

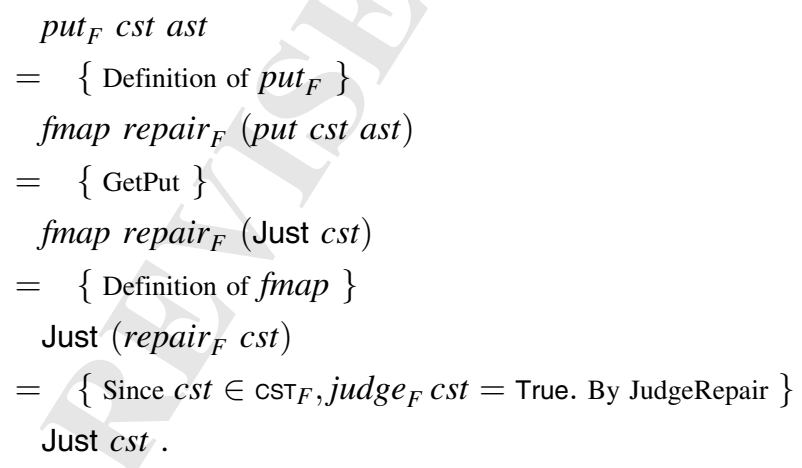

\section{Bi-Filter Directives}

847 Until now, we have only considered working with a single bi-filter, but this is 848 without loss of generality because we can provide a bi-filter composition operator 849 (Sect. 5.4.1) so that we can build large bi-filters from small ones. This is a

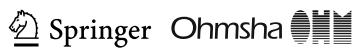

\begin{tabular}{|l|llll|}
\hline & Journal : Small-ext 354 & Dispatch : $\mathbf{1 3 - 2 - 2 0 2 0}$ & Pages : $\mathbf{5 5}$ \\
& Article No. : 82 & $\square$ LE & & $\square$ \\
MS Code : NGCO-D-19-00023R2 & $\square$ CP & TYPSET \\
\hline
\end{tabular}


850

851

852

853

854

855

856

857

858

859

860

861

862

863

864

865

866

867

89

871

872

873

874

875

suitable semantic foundation for introducing YACC-like directives for specifying priority and associativity into $\mathrm{BIYACC}_{\mathrm{A}}$ (Sect. 5.4.2), since we can give these directives a bi-filter semantics and interpret a collection of directives as the composition of their corresponding bi-filters. We will also discuss some properties related to this composition (Sect. 5.4.3).

\section{Bi-Filter Composition}

We start by defining bi-filter composition, with the intention of making the net effect of applying a sequence of bi-filters one by one the same as applying their composite. Although the intention is better captured by Lemma 6, which describes the repair and judge behaviour of a composite bi-filter in terms of the component bifilters, we give the definition of bi-filter composition first.

Definition 10 (Bi-filter composition) The composition of two bi-filters is defined by

$$
\begin{aligned}
(\triangleleft)::(t \rightarrow \text { Maybe } t) & \rightarrow(t \rightarrow \text { Maybe } t) \rightarrow(t \rightarrow \text { Maybe } t) \\
(j \triangleleft i) t=\text { case } i t \text { of } & \\
\text { Nothing } & \rightarrow j t \\
\text { Just } t^{\prime} & \rightarrow \text { case } j t^{\prime} \text { of } \\
\text { Nothing } & \rightarrow \text { Just } t^{\prime} \\
\text { Just } t^{\prime \prime} & \rightarrow \text { Just } t^{\prime \prime} .
\end{aligned}
$$

When applying a composite bi-filter $j \triangleleft i$ to a tree $t$, if $t$ is correct with respect to $i$ (i.e. $i t=$ Nothing), we directly pass the original tree $t$ to $j$; otherwise $t$ is repaired by $i$, yielding $t^{\prime}$, and we continue to use $j$ to repair $t^{\prime}$. Note that if $j t^{\prime}=$ Nothing, we return the tree $t^{\prime}$ instead of Nothing.

Lemma 6 For a composite bi-filter $j \triangleleft i$, the following two equations hold:

$$
\begin{gathered}
\text { repair }(j \triangleleft i) t=\left(\text { repair }_{j} \circ \text { repair }_{i}\right) t \\
\text { judge }(j \triangleleft i) t=j_{\text {udge }} t \wedge \text { judge }_{i} t .
\end{gathered}
$$

Proof By the definition of bi-filter composition.

Composition of bi-filters should still be a bi-filter and satisfy RepairJudge and PassThrough. This is not always the case though - to achieve this, we need some additional constraint on the component bi-filters, as formulated below.

Definition 11 Let $i$ and $j$ be bi-filters. We say that $j$ respects $i$ exactly when

$$
\text { judge }_{i} t=\text { True } \Rightarrow \text { judge }_{i}\left(\text { repair }_{j} t\right)=\text { True . }
$$

\begin{tabular}{|l|lllll|}
\hline & Journal : Small-ext 354 & Dispatch : 13-2-2020 & Pages : 55 \\
& Article No. : 82 & $\square$ LE & & $\square$ & TYPESET \\
& MS Code : NGCO-D-19-00023R2 & $\square$ CP & DISK \\
\hline
\end{tabular}


If $j$ respects $i$, then a later applied repair ${ }_{j}$ will never break what may already be repaired by a previous repair ${ }_{i}$. Thus in this case we can safely compose $j$ after $i$. This is proved as the following theorem.

Theorem 5 Let $i$ and $j$ be bi-filters (satisfying RepairJudge and PassThrough). If $j$ respects $i$, then $j \triangleleft i$ also satisfy RepairJudge and PassThrough.

Proof For RepairJudge, we reason:

$$
\begin{aligned}
& j u d g e(j \triangleleft i)(\text { repair }(j \triangleleft i) t) \\
= & \{\text { Lemma } 6\} \\
& j u d g e(j \triangleleft i)\left(\text { repair }_{j}\left(\text { repair }_{i} t\right)\right) \\
= & \{\text { Lemma } 6\} \\
& \text { judge }_{j}\left(\text { repair }_{j}\left(\text { repair }_{i} t\right)\right) \wedge \text { judge }_{i}\left(\text { repair }_{j}\left(\text { repair }_{i} t\right)\right) \\
= & \{\text { RepairJudge of } j\}\} \\
& \text { True } \wedge \text { judge }_{i}\left(\text { repair }_{j}\left(\text { repair }_{i} t\right)\right) \\
= & \left\{\text { judge }_{i}\left(\text { repair }_{i} t^{\prime}\right)=\text { True }_{j} \text { respects }_{i}\right\} \\
& \text { True } \wedge \text { True } \\
= & \text { True . }
\end{aligned}
$$

883 And for PassThrough:

$$
\begin{aligned}
& \operatorname{get}(\operatorname{repair}(j \triangleleft i) t) \\
& =\{\text { Lemma } 6\}
\end{aligned}
$$

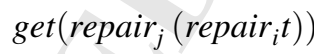

$$
\begin{aligned}
& =\{\text { PassThrough of } j\} \\
& \text { get }\left(\text { repair }_{i} t\right) \\
& =\{\text { PassThrough of } i\} \\
& \text { gett . }
\end{aligned}
$$

\section{Priority and Associativity Directives}

887 To relieve the burden of writing bi-filters manually and guaranteeing respect among 888 bi-filters being composed, we provide some directives for constructing bi-filters 889 dealing with priority ${ }^{7}$ and associativity, which are generally comparable to YACC's 890 conventional disambiguation directives. The bi-filter directives in a BIYACC program 891 can be thought of as specifying 'production priority tables', analogous to the operator precedence tables of, for example, the C programming language [24]

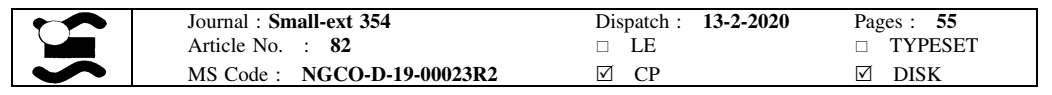


893

894

895

896

897

898

899

900

901

902

903

904

905

906

907

908

909

912

913

914

915

(chapter Expressions) and HASKELL [34] (page 51). The main differences (in terms of the parsing direction) are as follows:

- For bi-filters, priority can be assigned independently of associativity and vice versa, while the YACC-style approach does not permit so-by design, when the YACC directives (\%left, \%right, and \%nonassoc) are used on multiple tokens, they necessarily specify both the precedence and associativity of those tokens.

- For bi-filters, priority and associativity directives may be used to specify more than one production priority tables, making it possible to put unrelated operators in different tables and avoid (unnecessarily) specifying the relationship between them. It is impossible to do so with the YACC-style approach, for its concise syntax only allows a single operator precedence table.

(The bi-filter semantics of) our bi-filter directives repair CSTs violating priority and associativity constraints by adding parentheses-for example, if the production of addition expressions in Fig. 6 is left-associative, then we can repair $\sharp$ Plus 1 (Plus 2 3 ) by adding parentheses around the right subtree, yielding $\sharp$ Plus 1 (Paren (Plus 2 3)), provided that the grammar has a production of parentheses annotated with the bracket attribute $[8,53]$ :

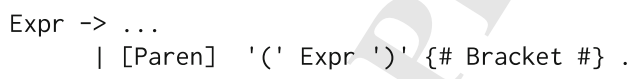

It instructs our bi-filter directives to use this production when parentheses need to be added. Internally, from the production and bracket attribute annotation, a type class AddParen and corresponding instances for each data type generated from concrete syntax (Expr for this example) are automatically created:

$$
\begin{gathered}
\text { class AddParen t where } \\
\text { canAddPar }:: \mathrm{t} \rightarrow \text { Bool } \\
\text { addPar }:: \mathrm{t} \rightarrow \mathrm{t}
\end{gathered}
$$

917 where canAddPar tells whether a CST can be wrapped in a parenthesis structure and 918 addPar adds that structure if it is possible or behaves as an identity function 919 otherwise. This makes it possible to automatically generate bi-filters to repair 920 incorrect CSTs (and help the user to define their own bi-filters more easily-see 921 Sect. 5.5).

922 In order for bi-filter directives to work correctly, the user should notice the 923 following requirements: (1) directives shall not mention the parenthesis production 924 annotated with bracket attribute so that they respect each other and work properly 925 (as introduced in Definition 11). (2) Suppose that the parenthesis production is $926 N T \rightarrow \alpha N T_{R} \beta$ where $\alpha$ and $\beta$ denote a sequence of terminals and $N T_{R}$ is a possibly 927 different nonterminal from $N T$ (on the right-hand side of the production)-for 928 instance, Expr $\rightarrow$ '('Expr')' above - there shall be exactly one printing action 929 defined for the parenthesis production in the form of $v+>\alpha\left[v+>N T_{R}\right] \beta$ for the 930 PassThrough property to hold: for any CST, the (added) parenthesis structure will 931 all be dropped through the conversion to its AST.

\section{包 Springer Ohmsha 率请}

\begin{tabular}{|l|lllll|}
\hline & Journal : Small-ext 354 & Dispatch : $\mathbf{1 3 - 2 - 2 0 2 0}$ & Pages : 55 \\
Article No. : 82 & $\square$ & LE & & TYPESET \\
& MS Code : NGCO-D-19-00023R2 & $\nabla$ & CP & $\square$ & DISK \\
\hline
\end{tabular}


Next we introduce our priority and associativity directives and their bi-filter semantics. From a directive, we first generate a bi-filter that checks and repairs only the top of a tree; this bi-filter is then lifted to check and repair all the subtrees in a tree. In the following, we will give the semantics of the directives in terms of the generation of the top-level bi-filters, and then discuss the lifted bi-filters and other important properties they satisfy in Sect. 5.4.3.

\section{Priority Directives}

A priority directive defines relative priority between two productions; it removes (in the parsing direction) or repairs (in the printing direction) CSTs in which a node of lower priority is a direct child of the node of higher priority. For instance, we can define that (the production of) multiplication has higher priority than (the production of) addition for the grammar in Fig. 6 by writing

$$
\begin{aligned}
& \text { Expr } \rightarrow \text { Expr ‘ } * \text { ' Expr }>\text { Expr } \rightarrow \text { Expr ' }+ \text { ' Expr } ; \\
& \text { or just Times }>\text { Plus; . }
\end{aligned}
$$

The directive first produces the following top-level bi-filter: ${ }^{8}$

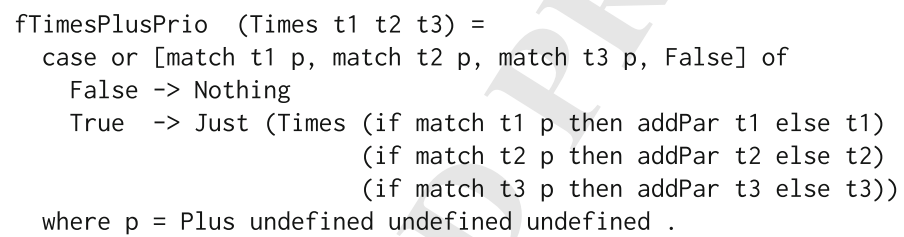

We first check whether any of the subtrees $\mathrm{t} 1, \mathrm{t} 2$, and $\mathrm{t} 3$ violates the priority constraint, i.e. having Plus as its top-level constructor-this is checked by the match function, which compares the top-level constructors of its two arguments. The resulting boolean values are aggregated using the list version of logical disjunction or $::[\mathrm{Bool}] \rightarrow$ Bool. If there is any incorrect part, we repair it by inserting a parenthesis structure using addPar.

In general, the syntax of priority directives is

$$
\begin{aligned}
& \text { Priority }::= \text { 'Priority:' PDirective } \\
& \text { PDirective }::=\text { ProdOrCons '>' ProdOrCons '; } \mid \text { ProdOrCons '<' ProdOrCons '; } \\
& \text { ProdOrCons }::=\text { Prod } \mid \text { Constructor } \\
& \text { Prod }::=\text { Nonterminal '->' Symbol }{ }^{+}
\end{aligned}
$$

where Constructor and Symbol are already defined in Fig. 4; for each priority declaration, we can use either productions or their names (i.e. constructors).

\footnotetext{
${ }^{8}$ Although terminals such as "*, and ' + ' are uniquely determined by constructors and not explicitly included in the CSTs, there are fields in CSTs for holding whitespaces after them. Thus Times still has three subtrees. Also, for simplicity, the bi-filter fTimesPlusPrio attempts to repair the whitespace subtree $t 2$ even though the repair can never happen since $t 2$ cannot match $p$.
}

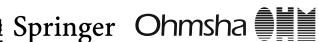

\begin{tabular}{|l|lllll|}
\hline & Journal : Small-ext 354 & Dispatch : & $\mathbf{1 3 - 2 - 2 0 2 0}$ & Pages : $\mathbf{5 5}$ \\
Article No. : $\mathbf{8 2}$ & $\square$ LE & TYPESET \\
\hline
\end{tabular}


If the user declares that a production $N T_{1} \rightarrow R H S_{1}$ has higher priority than another production $N T_{2} \rightarrow R H S_{2}$, the following priority bi-filter will be generated:

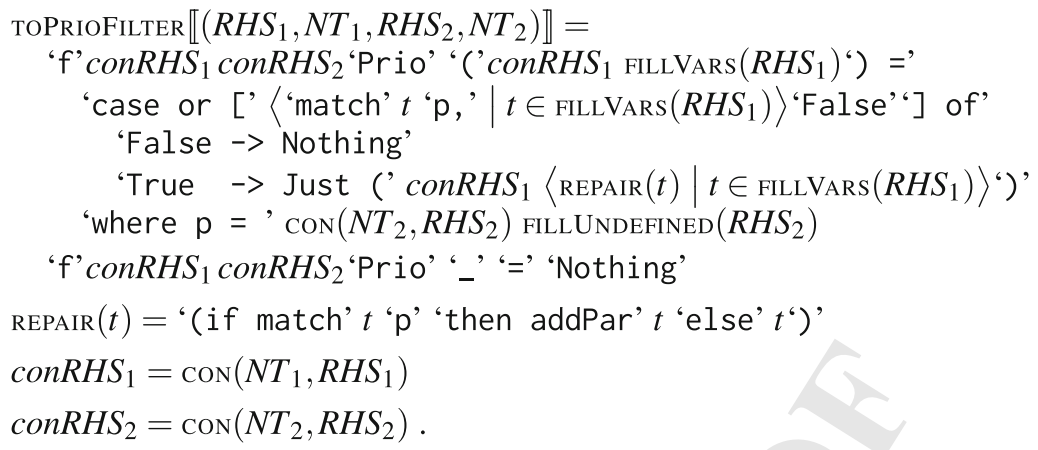

CON looks up constructor names for input productions (divided into nonterminals

961

962

963

964

965

966

967

969

970

971

972

973

974

975

976

977

978

979

and right-hand sides); FILLVARS(nt) generates variable names for each terminal and nonterminal in $n t$ (here $R H S_{1}$ ); FILLUNDEFINED is similar to FILLV $V_{\text {ARS }}$ but it produces undefined values instead. If productions are referred to using their constructors, we can simply look up the nonterminals and right-hand sides and use the same code generation strategy.

Transitive closures In the same way as conventional YACC-style approaches, the priority directives are considered transitive. For instance,

$$
\begin{aligned}
& \text { Expr } \rightarrow \text { Expr '*' Expr }>\text { Expr } \rightarrow \text { Expr '+' Expr; } \\
& \text { Expr } \rightarrow \text { Expr '+' Expr }>\text { Expr } \rightarrow \text { Expr '\&' Expr; }
\end{aligned}
$$

implies that Expr $\rightarrow$ Expr ' $*$ ' Expr $>$ Expr $\rightarrow$ Expr ' $\&$ ' Expr;. The feature is important in practice since it greatly reduces the amount of routine code the user needs to write (for large grammars).

\section{Associativity Directives}

Associativity directives assign (left- or right-) associativity to productions. A leftassociativity directive bans (or repairs, in the printing direction) CSTs having the pattern in which a parent and its right-most subtree are both left-associative, if the (relative) priority between the parent and the subtree is not defined; a rightassociativity directive works symmetrically.

As an example, we can declare that both addition and subtraction are leftassociative (for the grammar in Fig. 6) by writing

$$
\text { Left:Expr } \rightarrow \text { Expr '+' Expr, Expr } \rightarrow \text { Expr '-' Expr; }
$$

or just Left: Plus, Minus;. Since the relative priority between Plus and Minus is not defined, we generate top-level bi-filters for all the four possible pairs formed out of Plus and Minus:

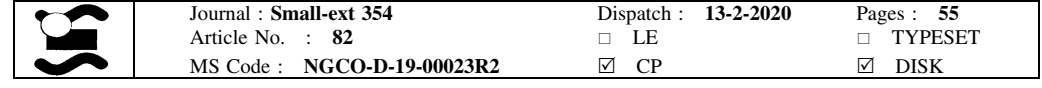




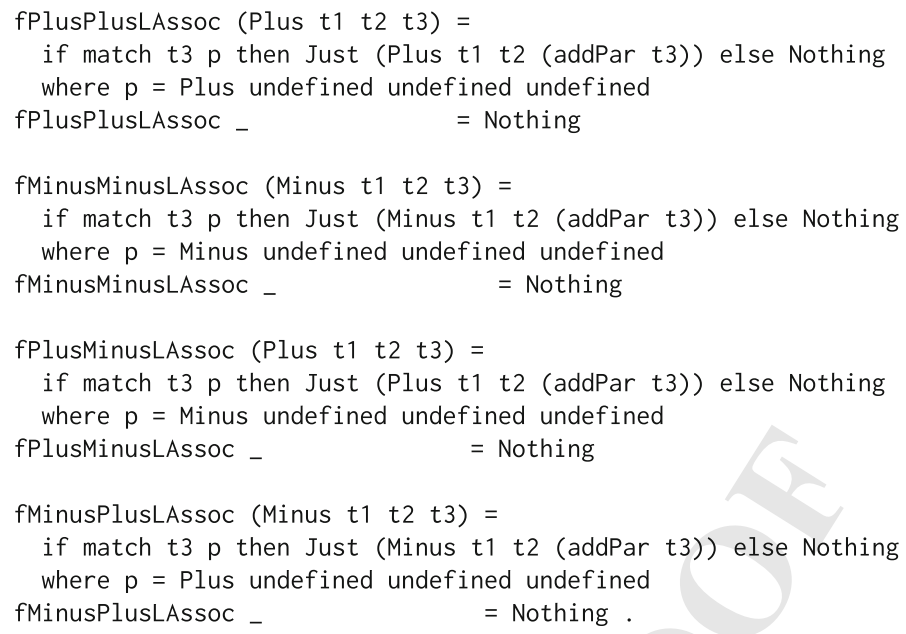

986 For instance, fPlusPlusLAssoc accepts \#Plus (Plus 12 2) 3 but not $987 \sharp_{\text {Plus }} 1$ (Plus 23 ), which is repaired to $\sharp_{\text {Plus }} 1$ (Paren (Plus 23 )).

988 Generally, the syntax of associativity directives is

$$
\begin{aligned}
\text { Associativity }::=\text { 'Associativity:' LeftAssoc RightAssoc } \\
\text { LeftAssoc }::=\text { 'Left:' ProdOrCons }{ }^{+}\left\{{ }^{\prime},\right\} \text { ';', } \\
\text { RightAssoc }::=\text { 'Right:'ProdOrCons }{ }^{+}\left\{{ }^{\prime},\right\} \text { ';' . }
\end{aligned}
$$

990 Now we explain the generation of (top-level) bi-filters from associativity directives. 991 We will consider only left-associativity directives, as right-associativity directives 992 are symmetric. For every pair of left-associative productions whose relative priority 993 is not defined-including cases where the two productions are the same-we 994 generate a bi-filter to repair CSTs whose top uses the first production and whose 995 right-most child uses the second production. Let $N T_{1} \rightarrow \alpha_{1} N T_{1 R}$ and $N T_{2} \rightarrow$ $996 \alpha_{2} N T_{2 R}$ be two such productions, where $\alpha_{1}\left(\alpha_{2}\right)$ matches a sequence of arbitrary 997 symbols of any length and $N T_{1 R}\left(N T_{2 R}\right)$ is the right-most symbol and must be a 998 nonterminal. (If it is not a nonterminal, it is meaningless to discuss associativity.) 999 The generated bi-filter is

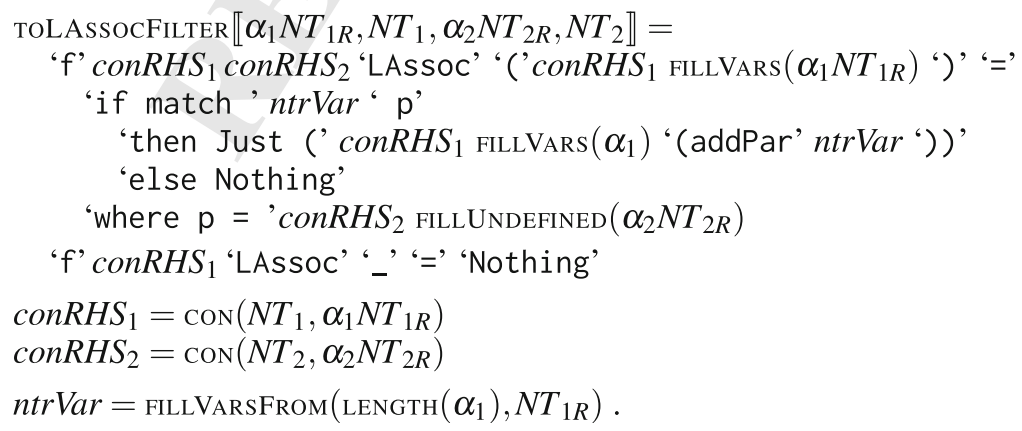

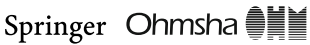

\begin{tabular}{lllll} 
Journal : Small-ext 354 & Dispatch : & $\mathbf{1 3 - 2 - 2 0 2 0}$ & \multicolumn{2}{c}{ Pages : $\mathbf{5 5}$} \\
Article No. $: \mathbf{8 2}$ & $\square$ LE & & $\square$ & TYPESET \\
MS Code : & NGCO-D-19-00023R2 & $\square$ & CP & $\square$ DISK \\
\hline
\end{tabular}


1002 Functions CON, FILLUNDEFINED, and FILLVAR have the same behaviour as before; 1003 FILLVARS - FROM (which is a variation of FILLVARS) generates variable names for each 1004 terminal and nonterminal in its argument with suffix integers counting from a given 1005 number to avoid name clashing.

1006

1007

1008

1009

Handling injective productions Sometimes the grammar may contain injective productions (also called chain productions) [9], which have only a single nonterminal on their right-hand side, like InfE $\rightarrow$ [FromE] Exp. When we use it to define a grammar

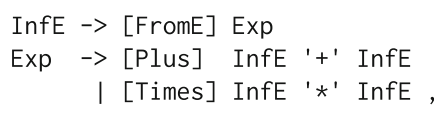

1011 program text $1+2{ }^{*} 3$ will be parsed to two CSTs, namely $\operatorname{cst}_{1}=\sharp_{\text {Plus }}($ FromE 1 ) and $1012 \operatorname{cst}_{2}=\sharp_{\text {Times }}($ FromE (Plus 12 2) (FromE 3)), and we want to spot (FromE (Times 23 3)) 1013 and discard it using the priority directive Times $>$ Plus. If handled naively, the bi1014 filter generated from the directive would only remove CSTs having pattern 1015 Times (Plus _ _ ) _ (and two other similar ones), but cst $_{2}$ would not match the pattern 1016 due to the presence of the FromE node between Times and Plus. We made some 1017 effort in the implementation to make the match function ignore the nodes 1018 corresponding to injective productions (FromE in this case).

\section{Properties of the Generated Bi-Filters}

1020 We discuss some properties of the bi-filters generated from our priority and associativity directives, to justify that it is safe to use these bi-filters without disrupting the well-behavedness of the whole system. Specifically:

- The generated top-level bi-filters satisfy RepairJudge, and it is easy to write actions to make them satisfy PassThrough.

- The bi-filters lifted from the top-level bi-filters still satisfy RepairJudge and PassThrough.

- The lifted bi-filters are commutative, which not only implies that all such bifilters respect each other and can be composed in any order, but also guarantees that we do not have to worry about the order of composition since it does not affect the behaviour.

We will give only high-level, even informal, arguments for these properties, since, due to the generic nature of the definitions of these bi-filters (in terms of Scrap Your Boilerplate [30]), to give formal proofs we would have to introduce rather complex machinery (e.g. datatype-generic induction), which would be tedious and distracting.

Top-level bi-filters The fact that the generated top-level bi-filters satisfy RepairJudge can be derived from the requirement that the directives do not mention the parenthesis production. Because of the requirement, in the generated bifilters, repairing is always triggered by matching a non-parenthesis production, and 1040 after that repairing will not be triggered again because a parenthesis production will 1041 have been added. For example, in the bi-filter fTimesPlusPrio (in Sect. 5.4.2), with

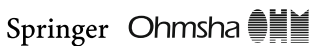

\begin{tabular}{|l|lllll|}
\hline & Journal : Small-ext 354 & Dispatch : $\mathbf{1 3 - 2 - 2 0 2 0}$ & Pages : 55 \\
& Article No. : 82 & $\square$ & LE & & $\square$ \\
MS Code : NGCO-D-19-00023R2 & $\square$ & CP & TYPESET \\
\hline
\end{tabular}


1042

1043

1044

1045

1046

1047

1048

1049

1050

1051

1052

1053

1055

1056

1057

1058

1059

1060

1061

1062

1063

1064

1065

1066

1067

1068

1069

1070

1071

1072

1073

1074

match $t 1 p$, match $t 2 p$, and match $t 3 p$ we check whether $t 1, t 2$, and $t 3$ has Plus as the top-level production, which is different from the parenthesis production Paren; if any of the matching succeeds, say $\mathrm{t} 1$, then addPar $\mathrm{t} 1$ will add Paren at the top of $\mathrm{t} 1$, and match (addPar t1) $p$ is guaranteed to be False, so the subsequent invocation of judge fTimesPlusPrio will return True. For PassThrough, since all the top-level bifilters do is add parenthesis productions, we can simply make sure that appearances of the parenthesis production are ignored by get, i.e. get (addPar $s)=$ get $s$ for all $s$; this, by well-behavedness, is the same as making put (printing actions) skip over parentheses. For example, for the grammar in Figure 6, we should write $\mathrm{t}+>$ ' (' $[\mathrm{t}+>$ Expr] ')' as the only printing action mentioning parentheses, which means that put (Paren $s) t=$ fmap Paren (put $s t$ ) for all $s$ and $t$. Then the following reasoning implies that get $($ Paren $s)=$ get $s$ for all $s$ :

$$
\begin{aligned}
& \text { get }(\text { Paren } s)=\text { Just } t \\
& \Leftrightarrow \quad\{\Rightarrow \text { by GetPut and } \Leftarrow \text { by PutGet }\} \\
& \quad \text { put }(\text { Paren } s) t=\text { Just }(\text { Paren } s) \\
& \Leftrightarrow \quad\{\text { By the above statement: put }(\text { Paren } s) t=\text { fmap Paren }(\text { put } s t)\} \\
& \text { fmap Paren }(\text { put } s t)=\text { Just }(\text { Paren } s) \\
& \Leftrightarrow \quad\{\text { Lemma } 5 \text { and the definition of fmap }\} \\
& \text { put } s t=\text { Just } s \\
& \Leftrightarrow \quad\{\Rightarrow \text { by PutGet and } \Leftarrow \text { by GetPut }\} \\
& \text { get } s=\text { Just } t
\end{aligned}
$$

for all $s$ and $t$.

Lifted bi-filters The lifted bi-filters apply the top-level bi-filters to all the subtrees in a CST in a bottom-up order. Formally, we can define, datatype-generically, a lifted bi-filter as a composition of top-level bi-filters, and use datatype-generic induction to prove that there is suitable respect among the top-level bi-filters being composed, and that the lifted bi-filter satisfies RepairJudge and PassThrough if the top-level ones do. But here we provide only an intuitive argument. What the lifted bi-filters do is find all prohibited pairs of adjoining productions and separate all the pairs by adding parenthesis productions. For RepairJudge, since all prohibited pairs are eliminated after repairing, there will be nothing left to be repaired in the resulting CST, which will therefore be deemed valid. For PassThrough, the intuition is the same as that for the top-level bi-filters.

Commutativity Composite bi-filters $i \triangleleft j$ and $j \triangleleft i$ may have different behaviours,

\begin{tabular}{|c|c|c|}
\hline Journal : Small-ext 354 & Dispatch: 13-2-2020 & Pages: $\mathbf{5 5}$ \\
\hline Article No. : $\mathbf{8 2}$ & $\square \quad \mathrm{LE}$ & $\square \quad$ TYPESET \\
\hline MS Code : NGCO-D-19-00023R2 & $\square \quad \mathrm{CP}$ & DISK \\
\hline
\end{tabular}
so in general we need to know the order of composition to figure out the exact behaviour of a composite bi-filter. This can be difficult when using our bi-filter directives, since a lot of bi-filters are implicitly generated from the directives, and it is not straightforward to specify the order in which all the explicitly and implicitly generated bi-filters are composed. Fortunately, we do not need to do so, for all the bi-filters generated from the directives are commutative, meaning that the order of composition does not affect the behaviour.

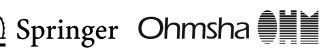


1075

1076

1079

1088

1081

1082

1083

1084

1085

1086

1087

1089

1090

1091

1092

1093

1094

1095

1096

1097

1098

1099

1100

1101

1102

1103

Definition 12 (Bi-filter commutativity) Two bi-filters $i$ and $j$ are commutative exactly when

$$
\text { repair }_{i} \circ \text { repair }_{j}=\text { repair }_{j} \circ \text { repair }_{i} .
$$

By Lemma 6, this implies repair $(i \triangleleft j)=$ repair $(j \triangleleft i)$. Note that judge $(i \triangleleft j)=j u d g e(j \triangleleft i)$ by definition, so we do not need to require this in the definition of commutativity.

An important fact is that commutativity is stronger than respect, so it is always safe to compose commutative bi-filters.

Lemma 7 Commutative bi-filters respect each other.

Proof Given commutative bi-filters $i$ and $j$, we show that $j$ respects $i$. Suppose that judge $_{i} t=$ True for a given tree $t$. Then

$$
\begin{aligned}
& \text { judge }_{i}\left(\text { repair }_{j} t\right) \\
= & \left\{\text { repair }_{i} t=t, \text { since }_{\text {judge }} t=\text { True }\right\} \\
& \text { judge }_{i}\left(\text { repair }_{j}\left(\text { repair }_{i} t\right)\right) \\
= & \{i \text { and } j \text { are commutative }\} \\
& \text { judge } \\
= & \text { repair } \left._{i}\left(\text { repair }_{j} t\right)\right) \\
= & \{\text { RepairJudge }
\end{aligned}
$$

It follows by symmetry that $i$ respects $j$ as well.

Now let us consider why any two different lifted bi-filters are commutative. (Commutativity is immediate if the two bi-filters are the same.) There are two key facts that lead to commutativity: (1) repairing does not introduce more prohibited pairs of productions, and (2) the prohibited pairs of adjoining productions checked and repaired by the two bi-filters are necessarily different. Therefore the two bifilters always repair different parts of a tree, and can repair the tree in any order without changing the final result. Fact (1) is, again, due to the requirement that the directives do not mention the parenthesis production, which is the only thing we add to a tree when repairing it. Fact (2) can be verified by a careful case analysis. For example, we might be worried about the situation where a left-associative directive looks for production $Q$ used at the right-most position under production $P$, while a priority directive also similarly looks for $Q$ used under $P$, but the two directives cannot coexist in the first place since the first directive implies $P$ and $Q$ have no relative priority whereas the second one implies $Q$ has lower priority than $P$.

\section{Manually Written Bi-Filters}

There are some other ambiguities that our directives cannot eliminate. In these cases, the user can define their own bi-filters and put them in the \#OtherFilters part in a BIYACC program as shown in Fig. 4. The syntax is

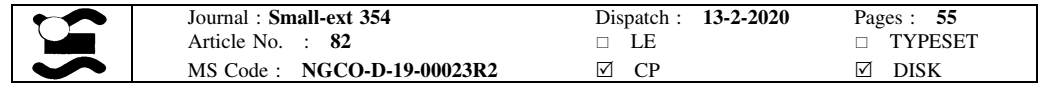




\section{OtherFilters $::=$ '['HsFunDecl ${ }^{+}\{$',' $\}$']' HsCode \\ HsFunDecl ::= HsFunName " : BiFilter' Nonterminal .}

1109 That is, this part of the program begins with a list of declarations of the names and 1110 types of the user-defined bi-filters, whose HASKELL definitions are then given below.

Now we demonstrate how to manually write a bi-filter by resolving the ambiguity brought by the dangling else problem. But before that, let us briefly review the problem, which arises, for example, in the following grammar:

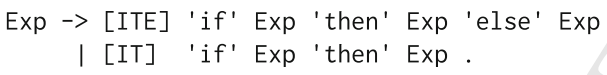

With respect to this grammar, the program text if a then if $x$ then $y$ else $z$ can be recognised as either if $a$ then (if $x$ then $y$ else $z$ ) or if a then (if $x$ then $y$ ) else $z$. To resolve the ambiguity, usually we prefer the 'nearest match' strategy (which is adopted by Pascal, C, and Java): else should match its nearest then, so that if a then (if $\mathrm{x}$ then $\mathrm{y}$ else $\mathrm{z}$ ) is the only correct interpretation.

The user may think that the problem can be solved by a priority (bi-)filter 1122 ITE > IT; in the hope that the production 'if-then-else' binds tighter than the 1123 production 'if-then'. Unfortunately, this is incorrect as pointed out by Klint and 1124 Visser [26], because the corresponding (bi-)filter incorrectly rules out the pattern

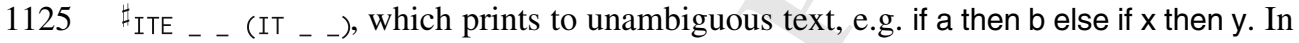
1126 fact, the (dangling else) problem is tougher than one might think and cannot be solved by any (bi-)filter performing pattern matching with a fixed depth [26].

Klint and Visser [26] proposed an idea to disambiguate the dangling-else grammar: let Greek letters $\alpha, \beta, \ldots$ match a sequence of symbols of any length. Then the program text if $\alpha$ then $\beta$ else $\gamma$ should be banned if the right spine of $\beta$ contains any if $\psi$ then $\omega$, as shown in the paper [26]. With the full power of (bi-)filters, which are fully fledged HASKELL functions, we can implement this solution in the following

1133 bi-filter:

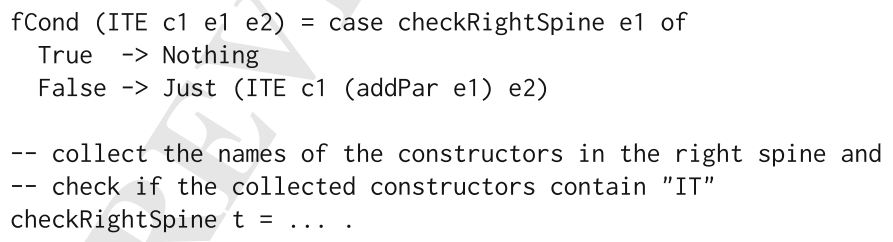

1135 This bi-filter is commutative with the bi-filters generated from our directives, 1136 since it (1) only searches for non-parenthesis productions that are not declared in 1137 any other directives, and (2) inserts only a parenthesis production when repairing

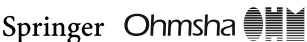

\begin{tabular}{lllll} 
Journal : Small-ext 354 & Dispatch : & $\mathbf{1 3 - 2 - 2 0 2 0}$ & \multicolumn{2}{l}{ Pages : $\mathbf{5 5}$} \\
Article No. : $\mathbf{8 2}$ & $\square \quad$ LE & & $\square$ & TYPESET \\
MS Code : NGCO-D-19-00023R2 & $\square$ & CP & $\square$ & DISK \\
\hline
\end{tabular}


incorrect CSTs. The reader may find the code of checkRightSpine in more detail in

1139 Fig. 10.

\section{Case Studies}

1141 The design of BIYACC may look simplistic and make the reader wonder how much it 1142 can describe. In fact, BIYACC can already handle real-world language features. For 1143 example, Kinoshita and Nakano [25] adopted BIYACC as part of their system for 1144 synchronising CoQ functions and corresponding OCAML programs. In this section, 1145 we demonstrate BIYACC with a medium-size case study: we use BIYACC to build a 1146 pair of parser and reflective printer for the TIGER language [4] and demonstrate some 1147 of their uses.

\section{The TIGER Language}

1149 TIGER is a statically typed imperative language first introduced in Appel's textbook 1150 on compiler construction [4]. Since TIGER's purpose of design is pedagogical, it is 1151 not too complex and yet covers many important language features including 1152 conditionals, loops, variable declarations and assignments, and function definitions 1153 and calls. TIGER is therefore a good case study with which we can test the potential 1154 of our BX-based approach to constructing parsers and reflective printers. Some of 1155 these features can be seen in this TIGER program:

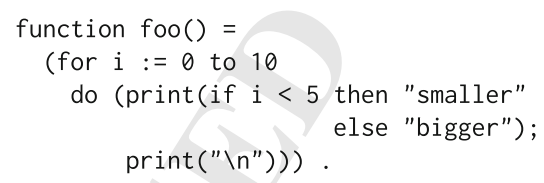

1157 To give a sense of Tiger's complexity, it takes a grammar with 81 production 1158 rules to specify Tiger's syntax, while for C89 and C99 it takes, respectively, 183 1159 and 237 rules without any disambiguation declarations (based on Kernighan and 1160 Ritchie [24] and the draft version of 1999 ISO C standard, excluding the 1161 preprocessing part). The difference is basically due to the fact that $\mathrm{C}$ has more 1162 primitive types and various kinds of assignment statements.

1163 Excerpts of the abstract and concrete syntax of TIGER are shown in Fig. 9. The 1164 abstract syntax is largely the same as the original one defined in Appel's textbook 1165 (page 98); as for the concrete syntax, Appel does not specify the whole grammar in 1166 detail, so we use a version slightly adapted from Hirzel and Rose's lecture notes 1167 [21]. Concretely, we add a parenthesis production to the grammar (and discard it 1168 when converting CSTs to ASTs, so that the PassThrough property could be 1169 satisfied), since TIGER's original grammar has no parenthesis production and an 1170 expression within round parentheses is regarded as a singleton expression sequence. 1171 This modification also makes it necessary to change the enclosing brackets for 1172 expression sequences from round brackets () to curly brackets \{\}, which helps 1173 (LALR(1) parsers) to distinguish a singleton expression sequence from an 1174 expression within parentheses. There is also another slight change in the definition

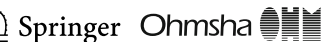

\begin{tabular}{|l|lllll|}
\hline & Journal : Small-ext 354 & Dispatch : $\mathbf{1 3 - 2 - 2 0 2 0}$ & Pages : 55 \\
& Article No. : 82 & $\square$ & LE & & TYPESET \\
& MS Code : NGCO-D-19-00023R2 & $\nabla$ & CP & $\square$ & DISK \\
\hline
\end{tabular}




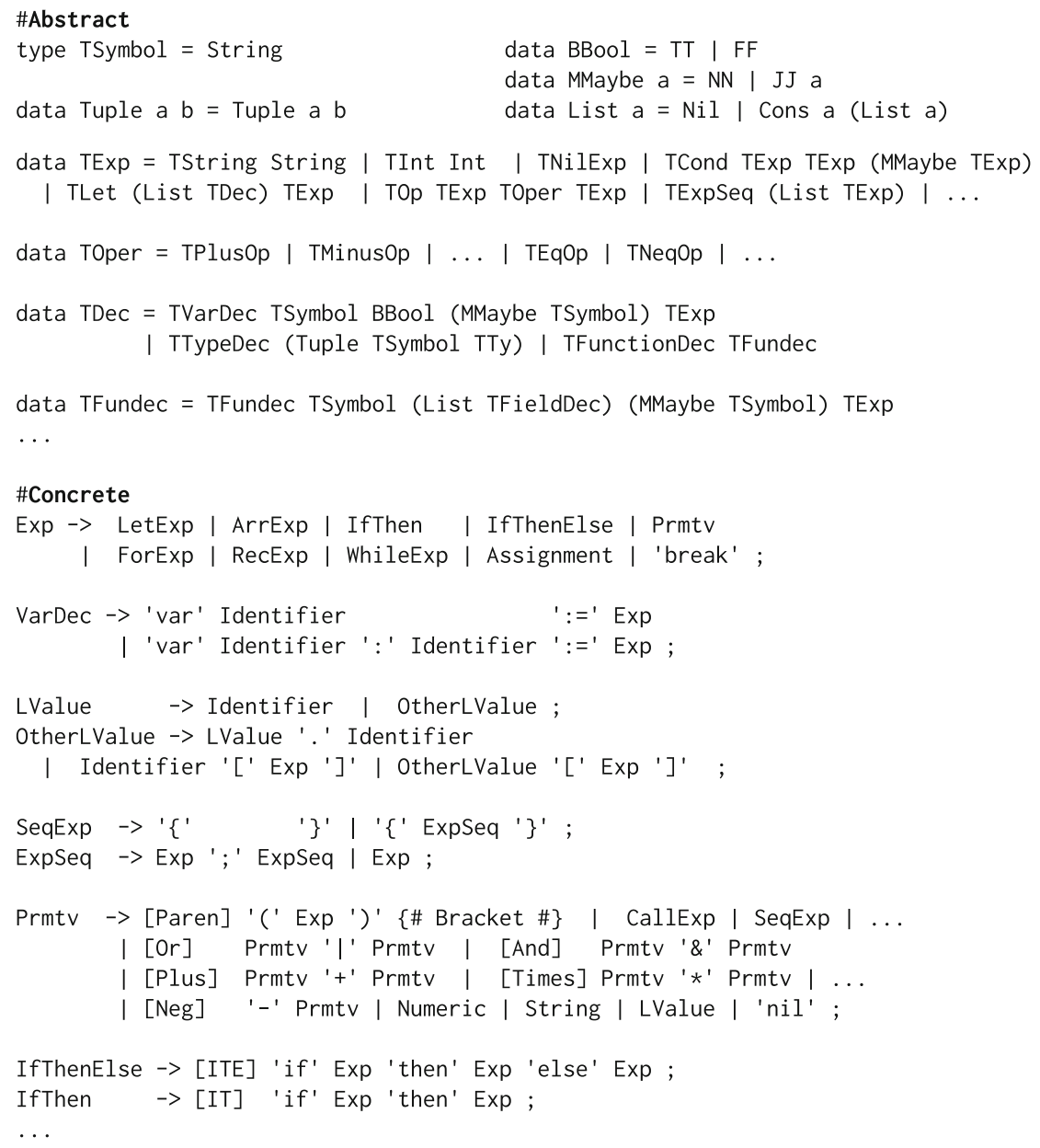

Fig. 9 An excerpt of Tiger's abstract and concrete syntax. (Here we define our own BBool type and MMaybe type to avoid name clashing with HASKELL's built-in ones)

1175 of ASTs for handling a feature not supported by the current BIYACC: the AST constructors TFunctionDec and TTypeDec take a single function or type declaration 1177 instead of a list of adjacent declarations (for representing mutual recursion) as in 1178 Appel [4], since we cannot handle the synchronisation between a list of lists (in 1179 ASTs) and a list (in CSTs) with BIYACC's current syntax.

1180 Following Hirzel and Rose's specification [21], the disambiguation directives for 1181 TIGER are shown in Fig. 10; for instance, we define multiplication to be left1182 associative. The directives also include a concrete treatment for the dangling else 1183 problem, which is usually 'not solved' when using a YACC-like (LA)LR parser 1184 generator to implement parsers: rather than resolving the grammatical ambiguity, 1185 we often rely on the default behaviour of the parser generator-preferring shift.

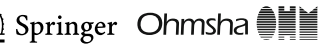

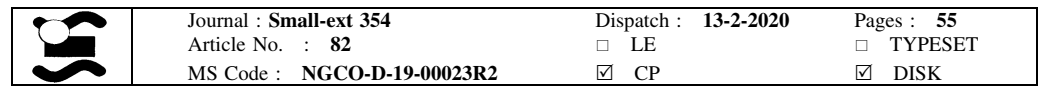




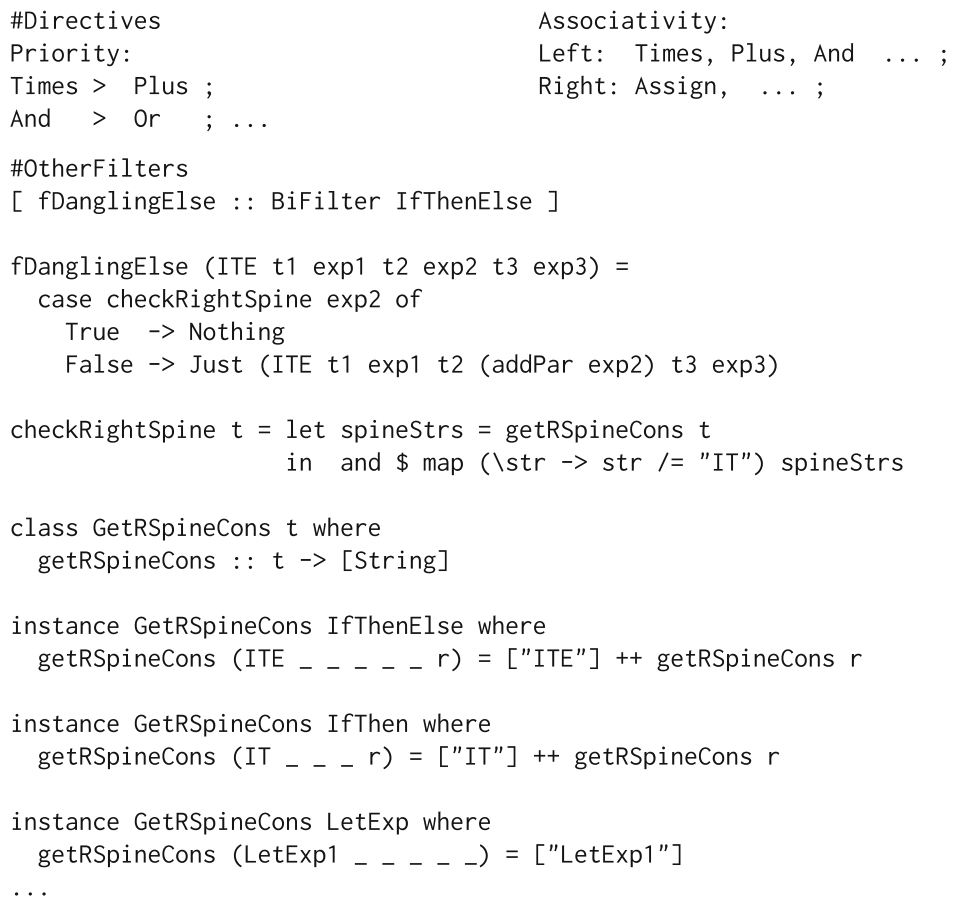

Fig. 10 An excerpt of the disambiguation directives for TIGER. (A type class GetRSpineCons is defined and implemented for collecting the constructors on the right spine of a given tree. Function getRSpineCons is recursively invoked for CSTs whose right-most subtree is (parsed from) a nonterminal)

1186 We have successfully tested our BIYACC program for TIGER on all the sample 1187 programs provided on the homepage of Appel's book, ${ }^{9}$ including a merge sort 1188 implementation and an eight-queen solver, and there is no problem parsing and 1189 printing them with well-behavedness guaranteed. In the following subsections, we 1190 will present some printing strategies described in the BIYACC program to 1191 demonstrate what BIYACC, in particular reflective printing, can achieve.

\section{Syntactic Sugar and Resugaring}

1193 We start with a simple example about syntactic sugar, which is pervasive in 1194 programming languages and lets the programmer use some features in an alternative 1195 (usually conceptually higher-level) syntax. For instance, TIGER represents boolean 1196 values false and true, respectively, as zero and nonzero integers, and the logical 1197 operators \& ('and') and | ('or') are converted to a conditional structure in the 1198 abstract syntax: e1 \& e2 is desugared and parsed to TCond e1 e2 (TInt 0) and e1 | e2 1199 to TCond e1 (TInt 1) e2. The printing actions for them in BIYACC are:

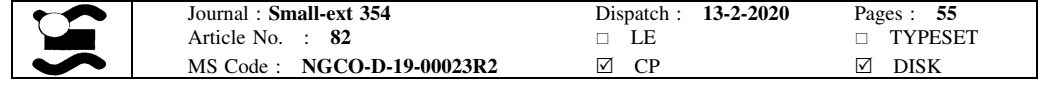




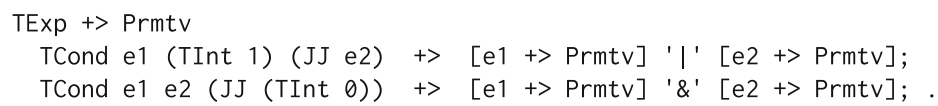

1215 which is parsed to inspecting the CST. source expression

A conventional printer which takes only the AST as input cannot reliably determine whether an abstract expression should be printed to the basic form or the sugared form, whereas a reflective printer can make the correct decision by

The idea of resugaring [42] is to print evaluation sequences in a core language in terms of a surface syntax. Here we show that, without any extension, BIYACC is already capable of propagating some AST changes that result from evaluation back to the concrete syntax, subsuming a part of Pombrio and Krishnamurthi's work [42, 43].

We borrow their example of resugaring evaluation sequences for the logical operators 'or' and 'not', but recast the example in TIGER. The 'or' operator has been defined as syntactic sugar in Section 6.2. For the 'not' operator, which Tiger lacks, we introduce ' $\sim$ ', represented by TNot in the abstract syntax. Now consider the

$$
\sim 1 \mid \sim \theta,
$$

$$
\text { TCond (TNot (TInt 1)) (TInt 1) (JJ (TNot (TInt 0))). }
$$

1217 A typical evaluator will produce the following evaluation sequence given the above 1218 AST:

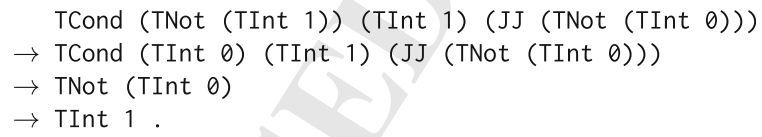

If we perform reflective printing after every evaluation step using BIYACC, we will get the following evaluation sequence on the source:

$\sim 1|\sim 0 \rightarrow 0| \sim 0 \rightarrow 0 \rightarrow 1$

Due to the PutGet property, parsing these concrete terms will yield the corresponding abstract terms in the abstract evaluation sequence, and this is exactly Pombrio and Krishnamurthi's 'emulation' property, which they have to prove for their system. For BIYACC, however, the emulation property holds by construction, since BIYACC programs are always well-behaved. Another difference is that we do not need to insert additional information (such as tags) into an AST for recording which surface syntax structure a node comes from. One advantage of our approach is that we keep the abstract syntax pure, so that other tools - the evaluator in particular-can process the abstract syntax without being modified, whereas in Pombrio and Krishnamurthi's approach, the evaluator has to be adapted to work on an enriched abstract syntax.

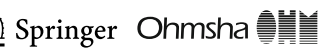

\begin{tabular}{lllll} 
Journal : Small-ext 354 & Dispatch : & $\mathbf{1 3 - 2 - 2 0 2 0}$ & \multicolumn{2}{c}{ Pages : $\mathbf{5 5}$} \\
Article No. $: \mathbf{8 2}$ & $\square$ LE & & $\square$ & TYPESET \\
MS Code : NGCO-D-19-00023R2 & $\square$ & CP & $\square$ DISK \\
\hline
\end{tabular}




\section{Language Evolution}

1235 When a language evolves, some new features of the language (e.g. the foreach loops 1236 introduced in Java 5 [20]) can be implemented by desugaring to some existing 1237 features (e.g. ordinary for loops), so that the compiler back-end and abstract syntax 1238 definition do not need to be extended to handle the new features. As a consequence, 1239 all the engineering work about optimising transformations or refactoring [18] that 1240 has been developed for the abstract syntax remains valid.

Consider a kind of 'generalised-if' expression allowing more than two cases, resembling the alternative construct in Dijkstra's guarded command language [12]. We extend Tiger's concrete syntax with the following production rules:

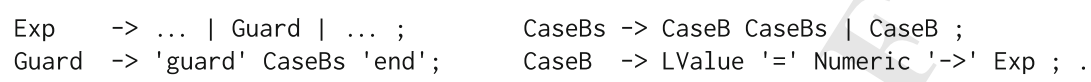

For simplicity, we restrict the predicate produced by CaseB to the form LValue '=' Numeric, but in general the Numeric part can be any expression computing an integer. The reflective printing actions for this new construct can still be written within BIYACC, but require much deeper pattern matching:

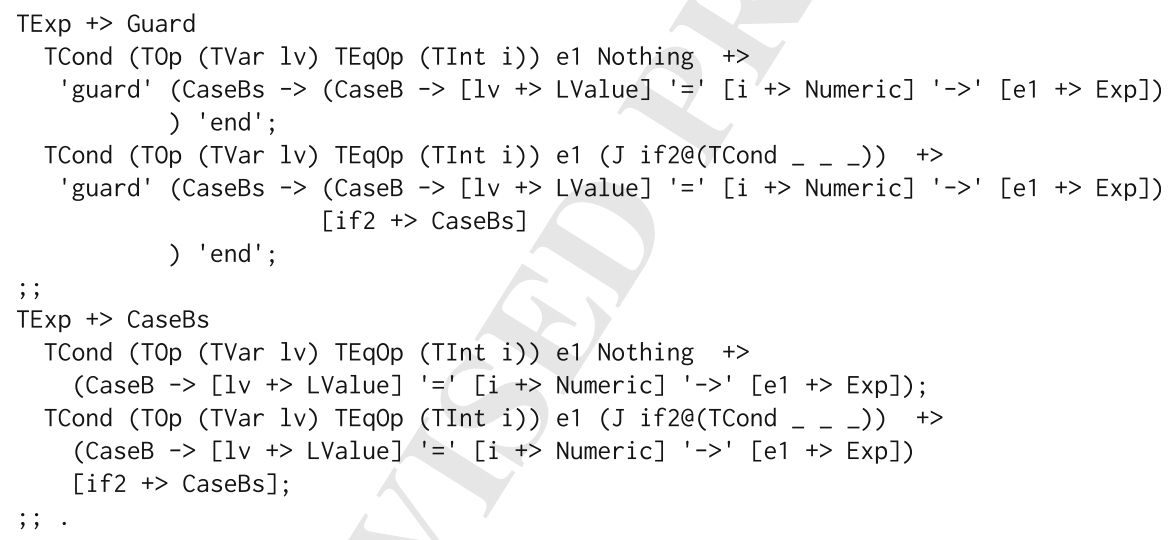

1252 Although being a little complex, these printing actions are in fact fairly 1253 straightforward: The first group of type Tiger $+>$ Guard handles the enclosing 1254 guard-end pairs, distinguishes between single- and multi-branch cases, and delegates 1255 the latter case to the second group, which prints a list of branches recursively.

1256 This is all we have to do-the corresponding parser is automatically derived and 1257 guaranteed to be consistent. Now guard expressions are desugared to nested if 1258 expressions in parsing and preserved in printing, and we can also resugar evaluation 1259 sequences on the ASTs to program text. For instance, the following guard expression

$$
\begin{aligned}
\text { guard } & \text { choice }=1->4 \\
\text { choice } & =2->8 \\
\text { choice } & =3 \rightarrow 16 \text { end }
\end{aligned}
$$

1261 is parsed to

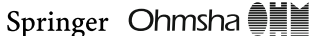

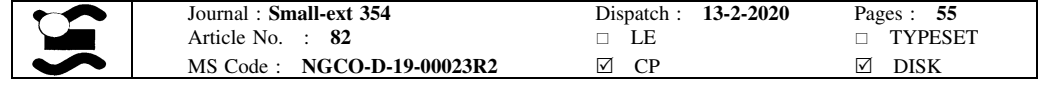


TCond (TOp (TVar (TSV "c")) TEqOp (TInt 1)) (TInt 4) (JJ

(TCond (TOp (TVar (TSV "c")) TEqOp (TInt 2)) (TInt 8) (JJ

(TCond (TOp (TVar (TSV "c")) TEqOp (TInt 3)) (TInt 16) NN))))

1263

1266

1265

1269

where TSimpleVar is shortened to TSV, and choice is shortened to c. Suppose that the value of the variable choice is 2 . The evaluation sequence on the AST will then be:

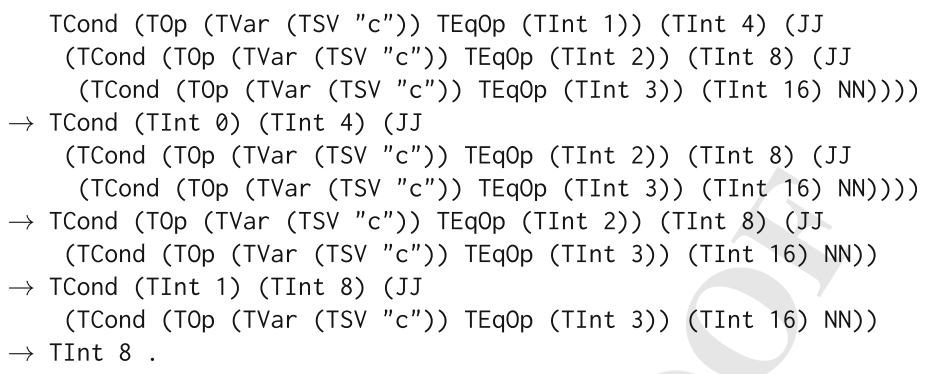

And the reflected evaluation sequence on the concrete expression will be:

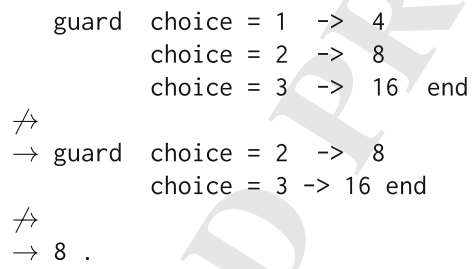

Reflective printing fails for the first and third steps (the program text becomes an if-then-else expression if we do printing at these steps), but this behaviour in fact conforms to Pombrio and Krishnamurthi's 'abstraction' property, which demands that core evaluation steps that make sense only in the core language must not be propagated to the surface. In our example, the first and third steps in the TCondsequence evaluate the condition to a constant, but conditions in guard expressions are restricted to a specific form and cannot be a constant; evaluation of guard expressions thus has to proceed in bigger steps, throwing away or going into a branch in each step, which corresponds to two steps for TCond.

The reader may have noticed that, after the guard expression is reduced to two branches, the layout of the second branch is disrupted; this is because the second branch is in fact printed from scratch. In current BIYACC, the printing from an AST to a CST is accomplished by recursively performing pattern matching on both tree structures. This approach naturally comes with the disadvantage that the matching is mainly decided by the position of the nodes in the AST and CST. Consequently, a minor structural change on the AST may completely disrupt the matching between the AST and the CST.

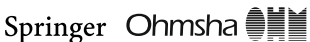

\begin{tabular}{lllll} 
Journal : Small-ext 354 & Dispatch : & $\mathbf{1 3 - 2 - 2 0 2 0}$ & \multicolumn{2}{c}{ Pages : $\mathbf{5 5}$} \\
Article No. $: \mathbf{8 2}$ & $\square$ LE & & $\square$ & TYPESET \\
MS Code : NGCO-D-19-00023R2 & $\square$ & CP & $\square$ & DISK \\
\hline
\end{tabular}


1291 We conclude this section by shortly discussing several other potential applications. 1292 In general, (current) BIYACC can easily and reliably propagate AST changes that 1293 have local effect such as replacing part of an AST with a simpler tree, without 1294 destroying the layouts and comments of unaffected code. Thus it would not be 1295 surprising that BIYACC can also propagate (1) simplification-like optimisations such 1296 as constant folding and constant propagation and (2) some code refactoring 1297 transformations such as variable renaming. All these functionalities are achieved for 1298 free by one 'general-purpose' BIYACC program, which does not need to be tailored 1299 for each application.

\section{Related Work}

\section{Unifying Parsing and Printing}

1302 Much research has been devoted to describing parsers and printers in a single 1303 program. For example, both Rendel and Ostermann [44] and Matsuda and Wang $1304[36,37]$ adopt a combinator-based approach ${ }^{10}$ (whereas we use a generator-based 1305 approach), where small components are glued together to yield more sophisticated 1306 behaviour, and can guarantee properties similar to Theorem 1 with cst replaced by 1307 ast in the equations. (Let us call the variant version Theorem 1' ${ }^{\prime}$, since it will be used 1308 quite often later.) In Rendel and Ostermann's system (called 'invertible syntax 1309 descriptions', which we shorten to ISDs henceforth), both the parsing and printing 1310 semantics are predefined in the combinators and consistency is guaranteed by their 1311 partial isomorphisms, whereas in Matsuda and Wang's system (called FuIPrR), the 1312 combinators describing pretty printing are translated by a semantic-preserving 1313 transformation to a core syntax, which is further processed by their grammar-based 1314 inversion system [38] to realise the parsing semantics. Brabrand et al. [7] present a 1315 tool XSugar that handles bijections between the XML syntax (representation) and 1316 any other syntax (representation) for the same language, guaranteeing that the 1317 syntax transformation is reversible. However, the essential factor that distinguishes 1318 our system from others is that the printer produced from a BIYACC program is reflective and can deal with synchronisation.

Although the above-mentioned systems are tailored for unifying parsing and printing, there are design differences. An ISD is more like a parser, while FuIPPR lets the user describe a printer: To handle operator priorities, for example, the user of ISDs will assign priorities to different operators, consume parentheses, and use combinators such as chainl to handle left recursion in parsing, while the user of FLIPPR will produce necessary parentheses according to the operator priorities. For basic BIYACC (that deals with unambiguous grammars only), the user defines a concrete syntax that has a hierarchical structure (e.g. Expr, Term, and Factor) to express operator priority, and write printing strategies to produce (preserve)

10FL01 ${ }^{10}$ Although they use different implementation techniques, we will not dive into them in our related work. 10FL02 See Matsuda and Wang's related work for a comparison [36].

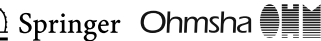

\begin{tabular}{|l|llll|}
\hline & Journal : Small-ext 354 & Dispatch : & $\mathbf{1 3 - 2 - 2 0 2 0}$ & Pages : 55 \\
Article No. : 82 & $\square \quad$ LE & $\square$ & TYPESET \\
& MS Code : NGCO-D-19-00023R2 & $\square \quad C P$ & $\square$ & DISK \\
\hline
\end{tabular}


necessary parentheses. The user of XSugar will also likely need to use such a hierarchical structure.

It is interesting to note that the part producing parentheses in FLIPPR essentially corresponds to the hierarchical structure of grammars. For example, to handle arithmetic expressions in FLIPPR, we can write:

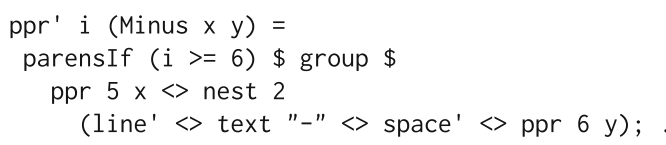

FLIPPR will automatically expand the definition and derive a group of $\mathrm{ppr}_{\mathrm{i}}$ functions indexed by the priority integer $i$, corresponding to the hierarchical grammar structure. In other words, there is no need to specify the concrete grammar, which is already implicitly embedded in the printer program. This makes FLIPPR programs neat and concise. Following this idea, BIYACC programs can also be made more concise: in a BIYACC program, the user is allowed to omit the production rules in the concrete syntax part (or omit the whole concrete syntax part), and they will be automatically generated by extracting the terminals and nonterminals in the righthand sides of all actions. However, if these production rules are supplied, BIYACC will perform some sanity checks: it will make sure that, in an action group, the user has covered all of the production rules of the nonterminal appearing in the 'type declaration', and never uses undefined production rules.

Just like basic BIYACC, all of the systems described above (aim to) handle unambiguous grammars only. Theoretically, when the user-defined grammar (or the derived grammar) is ambiguous, ISDs' partial isomorphism could guarantee Theorem $1^{\prime}$ by returning Nothing on ambiguous input; FLIPPR's (own) Theorem 1 is comparable to Theorem $1^{\prime}$ by taking all the language constructs which may cause non-injective printing into account. However, according to the paper, FLIPPR's Theorem 1 appears to only consider nondeterministic printing based on prettiness (layouts). Since the discussion on ambiguous grammars has not been presented in their papers, we tested their implementation and the behaviour is as follows: neither ISDs nor FLIPPR will notify the user that the (derived) grammar is ambiguous at compile time. For ISDs, the right-to-left direction of our Theorem $1^{\prime}$ will fail, while for FLIPPR, both directions will fail. (They never promise to handle ambiguous grammars, though.) In contrast, Brabrand et al. [7] give a detailed discussion about ambiguity detection, and XSugar statically checks if the transformations are 'reversible'. If any ambiguity in the program is detected, XSugar will notify the user of the precise location where ambiguity arises. In BIYACC, the ambiguity detection of the input grammar is performed by the employed parser generator (currently HAPPY), and the result is reported at compile time; if no warning is reported, the well-behavedness is always guaranteed. Note that the ambiguity detection can produce false positives: warnings only mean that the grammar is not LALR(1) but does not necessarily mean that the grammar is ambiguous - ambiguity detection is undecidable for the full CFG [10].

Here we also briefly discuss ambiguity detection for the filter approaches: priority and associativity (bi-)filters can be applied to (LA)LR parse tables to resolve (shift/

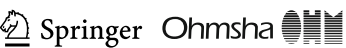

\begin{tabular}{lllll} 
Journal : Small-ext 354 & Dispatch : & $\mathbf{1 3 - 2 - 2 0 2 0}$ & \multicolumn{2}{c}{ Pages : $\mathbf{5 5}$} \\
Article No. $: \mathbf{8 2}$ & $\square$ LE & & $\square$ & TYPESET \\
MS Code : NGCO-D-19-00023R2 & $\square$ & CP & $\square$ DISK \\
\hline
\end{tabular}


1372

1373

1374

1375

1376

1377

1378

1379

1380

1381

1382

1383

1384

1385

1386

1387

1388

1389

1390

1391

1392

1393

1394

1395

1396

1397

1398

1399

1400

1401

1402

1403

1404

1405

1406

1407

1408

1409

1410

1411

1412

1413

1414

1415

reduce) conflicts $[9,26,52,53]$, and thus the completeness for simple (bi-)filters (see Definition 9) on LALR(1) grammars can be statically checked. However, our implementation does not support it, for bi-filter directives are more general, as stated in the beginning of Sect. 5.4.2, and therefore cannot be transformed to the underlying parser generator's YACC-style directives. Finding a way to directly apply priority and associativity bi-filters to parse tables (generated by HAPPY) is left as future work.

Finally, we compare BIYACC with an industrial tool, AugEAS, which provides the user with a local configuration API that converts configuration data into a rose tree representation [31]. Similar to BIYACC, Augeas also uses the idea of state-based asymmetric lenses so that its parse and print functions satisfy well-behavedness and it tries to preserve comments and layouts when printing the tree representation back. However, since the purpose of AugEAS and BIYACC is different, the differences between the tools are also noticeable: (1) AUGEAS works for regular grammars while BIYACC works for (unambiguous) context-free grammars. (2) Augeas uses a combinator-based approach while BIYACC adopts a generator-based approach. (3) AugEAS works more like a simple parser that stops after constructing CSTs: in the parsing direction, AugEas unambiguously separates strings into sub-strings, turn sub-strings into tokens, and use tokens to build the corresponding tree; but since each lens combinator (of AugEas) has its predefined strategy to turn its acceptable strings into the tree representation, the corresponding tree will be determined once the input string and the lens combinators for parsing the string are given; Augeas does not provide a functionality to further transform a tree. On the other hand, BIYACC first turns a string into its isomorphic CST (fully determined the input string and the grammar description) and finally converts the CST to its AST in accordance with the algebraic data types defined by the user; that is, the relation between a string (CST) and its AST is not predetermined but can be adjusted by the user (through printing actions).

\section{Generalised Parsing, Disambiguation, and Filters}

The grammar of a programming language is usually designed to be unambiguous. Various parser-dependent disambiguation methods such as grammar transformation [29] and parse table conflicts elimination [23] have been developed to guide the parser to produce a single correct CST [26]. On the other hand, natural languages that are inherently ambiguous usually require their parsing algorithms to produce all the possible CSTs; this requirement gives rise to algorithms such as Earley [14] and generalised LR [50] (GLR for short). Although these parsing algorithms produce all the possible CSTs, both their time complexity and space complexity are reasonable. For instance, GLR runs in cubic time in the worst situation and in linear time if the grammar is 'almost unambiguous' [48].

The idea to relate generalised parsing with parser-independent disambiguation for programming languages is proposed by Klint and Visser [26]. They proposed two classes of filters, property filters (defined in terms of predicates on a single tree) and comparison filters (defined in terms of relations among trees), but we only adapt and bidirectionalise predicate filters in this paper. One difficulty lies in the fact that

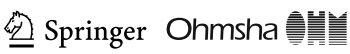

\begin{tabular}{|l|lllll|}
\hline & Journal : Small-ext 354 & Dispatch : 13-2-2020 & Pages : 55 \\
Article No. : 82 & $\square$ LE & $\square$ & TYPESET \\
& MS Code : NGCO-D-19-00023R2 & $\square$ CP & $\square$ & DISK \\
\hline
\end{tabular}


it is unclear how to define repair for comparison filters, as they generally select better trees rather than absolutely correct ones - in the printing direction, since put only produces a single CST, we do not know whether this CST needs repairing or not (for there is no other CST to compare). This is also one of the most important problems for our future work.

Parser-independent disambiguation (for handling priority and associativity conflicts) can also be found in LaLonde and des Rivieres's [29] and Aasa's [1] work. At first glance, our repair function is quite similar to LaLonde and des Rivieres's post-parse tree transformations that bring a CST into an expression tree, on whose nodes additional restrictions of priority and associativity are imposed. To be simple (but not completely precise), a CST's corresponding expression tree is obtained by first dropping all the nodes constructed from injective productions ${ }^{11}$ (note that parentheses nodes are still kept) and then use a precedence-introducing tree transformation to reshape the result. The transformation will do 'repairing' by rotating all the adjacent nodes of the tree where priority or associativity constraint is violated. By contrast, our repair function is simpler and only introduces parentheses in places where the judge function returns False. In short, their tree transformations are a kind of parser-independent disambiguation which does not require generalised parsing; however, those tree transformations are (almost) not applicable in the printing direction if well-behavedness is taken into consideration (due to the rotation of CSTs). Furthermore, it is not clear whether their approach can be generalised to handle other types of conflicts rather than the ones caused by priority and associativity.

There is much research on how to handle ambiguity in the parsing direction as discussed above; conversely, little research is conducted for 'handling ambiguity in the printing direction' and we find only one paper [8] that describes how to produce correct program text regarding priority and associativity, which is also one of the bases of our work. We extend their work [8] by allowing the bracket attribute to work with injective productions such as $\mathrm{E} \rightarrow \mathrm{T} ; \mathrm{T} \rightarrow \mathrm{F} ; \mathrm{F} \rightarrow>$ ' (' $\mathrm{E}$ ') ' \# Bracket\#;. (The previous work seems to only support the bracket attribute in the form of $\mathrm{E} \rightarrow$ ' '(' E ')' \# Bracket \#; ; whether the nonterminal E on the left-hand side and righthand side can be different is not made clear.)

Finally, we compare our approach with the conventional ones in general. In history, a printer is believed to be much simpler than a parser and is usually developed independently (of its corresponding parser). While a few printers choose to produce parentheses at every occasion naively, most of them take disambiguation information (for example, from the language's operator precedence table) into account and try to produce necessary parentheses only. However, as the YACC-style conventional disambiguation [23] is parser-dependent, this parentheses-adding technique is also printer-dependent. As the post-parse disambiguation increases the modularity of the (front-end of the) compiler [29], we believe that our post-print parentheses-adding increases the modularity once again. Additionally, the unification of disambiguation for both parsing and printing makes it possible for us to

11 An injective production, or a chain production, is one whose right-hand side is a single nonterminal; for instance, $\mathrm{E} \rightarrow \mathrm{N}$.

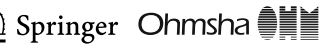

\begin{tabular}{lllll} 
Journal : Small-ext 354 & Dispatch : & $\mathbf{1 3 - 2 - 2 0 2 0}$ & \multicolumn{2}{l}{ Pages : $\mathbf{5 5}$} \\
Article No. $: \mathbf{8 2}$ & $\square \quad$ LE & & $\square \quad$ TYPESET \\
MS Code : NGCO-D-19-00023R2 & $\square \quad C P$ & $\square \quad$ DISK \\
\hline
\end{tabular}


1459 impose bi-filter laws, which further makes it possible to guarantee the well-

1460 behavedness of the whole system.

\section{Comparison with a Get-Based Approach}

1462 Our work is theoretically based on asymmetric lenses [17] of bidirectional 1463 transformations [11, 19], particularly taking inspiration from the recent progress on 1464 putback-based bidirectional programming [15, 27, 28, 40, 41]. As explained in 1465 Sect. 3, the purpose of bidirectional programming is to relieve the burden of 1466 thinking bidirectionally - the programmer writes a program in only one direction, 1467 and a program in the other direction is derived automatically. We call a language 1468 get-based when programs written in the language denote get functions, and call a 1469 language putback-based when its programs denote put functions. In the context of 1470 parsing and reflecting printing, the get-based approach lets the programmer describe 1471 a parser, whereas the putback-based approach lets the programmer describe a 1472 printer. Below we discuss in more depth how the putback-based methodology 1473 affects BIYACC's design by comparing BIYACC with a closely related, get-based 1474 system.

1475 Martins et al. [35] introduces an attribute grammar-based BX system for defining 1476 transformations between two representations of languages (two grammars). The 1477 utilisation is similar to BIYACC: The programmer defines both grammars and a set of 1478 rules specifying a forward transformation (i.e. get), with a backward transformation 1479 (i.e. put) being automatically generated. For example, the BIYACC actions in lines $1480 \quad 28-30$ of Fig. 2 can be expressed in Martins et al.'s system as

$$
\begin{aligned}
& \operatorname{get}_{A}^{E}\left(p l u s\left(x,{ }^{\prime}+, y\right)\right) \rightarrow \operatorname{add}\left(\operatorname{get}_{A}^{E}(x), \operatorname{get}_{A}^{T}(y)\right) \\
& \operatorname{get}_{A}^{E}\left(\operatorname{minus}\left(x,{ }^{\prime}-,, y\right)\right) \rightarrow \operatorname{sub}\left(\operatorname{get}_{A}^{E}(x), \operatorname{get}_{A}^{T}(y)\right) \\
& \operatorname{get}_{A}^{E}(e t(e)) \quad \longrightarrow \operatorname{get}_{A}^{T}(e)
\end{aligned}
$$

1482 which describes how to convert certain forms of CSTs to corresponding ASTs. The 1483 similarity is evident, and raises the question as to how get-based and putback-based 1484 approaches differ in the context of parsing and reflective printing.

1485 The difference lies in the fact that, with a get-based system, certain decisions on 1486 the backward transformation are, by design, permanently encoded in the bidirec1487 tionalisation system and cannot be controlled by the user, whereas a putback-based 1488 system can give the user fuller control. For example, when no source is given and 1489 more than one rule can be applied, Martins et al.'s system chooses, by design, the 1490 one that creates the most specialised version. This might or might not be ideal for 1491 the user of the system. For example: suppose that we port to Martins et al.'s system 1492 the BIYACC action that relates TIGER's concrete ' $\&$ ' operator with a specialised 1493 abstract if expression in Sect. 6.2, coexisting with a more general rule that maps a 1494 concrete if expression to an abstract if expression. Then printing the AST TCond 1495 (TSV "a") (TSV "b") 0 from scratch will and can only produce $a$ \& b, as dictated by the 1496 system's hard-wired printing logic. By contrast, the user of BIYACC can easily 1497 choose to print the AST from scratch as a \& b or if a then b else 0 by suitably 1498 ordering the printing actions.

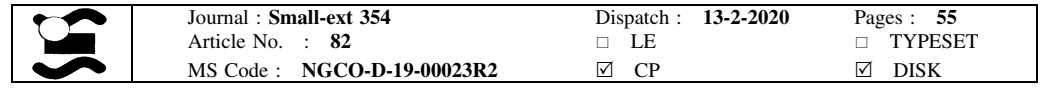


This difference is somewhat subtle, and one might argue that Martins et al.'s design simply went one step too far-if their system had been designed to respect the rule ordering as specified by the user, as opposed to always choosing the most specialised rule, the system would have given its user the same flexibility as BIYACC. Interestingly, whether to let user-specified rule/action ordering affect the system's behaviour is, in this case, exactly the line between get-based and putbackbased design. The user of Martins et al.'s system writes rules to specify a forward transformation, whose semantics is the same regardless of how the rules are ordered, and thus it would be unpleasantly surprising if the rule ordering turned out to affect the system's behaviour. By contrast, the user of BIYACC only needs to think in one direction about the printing behaviour, for which it is natural to consider how the actions should be ordered when an AST has many corresponding CSTs; the parsing behaviour will then be automatically and uniquely determined. In short, relevance of action ordering is incompatible with get-based design, but is a natural consequence of putback-based thinking.

\section{Conclusion}

We conclude the paper by summarising our contributions:

- We have presented the design and implementation of BIYACC, with which the programmer can describe both a parser and a reflective printer for a fully disambiguated context-free grammar in a single program. Our solution guarantees the partial version of the consistency properties (Definition 2) by construction.

- We proposed the notion of bi-filters, which enables BIYACC to disambiguate ambiguous grammars while still respecting the consistency properties. This is the main new contribution compared to the previous SLE'16 version [55].

- We have demonstrated that BIYACC can support various tasks of language engineering, from traditional constructions of basic machinery such as printers and parsers to more complex tasks such as resugaring, simple refactoring, and language evolution.

Acknowledgements We thank the reviewers and the editor for their selflessness and effort spent on reviewing our paper, a quite long one. With their help, the readability of the paper is much improved, especially regarding how several case studies are structured, how theorems for the basic BIYACC and theorems for the extended version handling ambiguous grammars are related, and how look-alike notions are 'disambiguated'. This work is partially supported by the Japan Society for the Promotion of Science (JSPS) Grant-in-Aid for Scientific Research (S) No. 17H06099; in particular, most of the second author's contributions were made when he worked at the National Institute of Informatics and funded by the Grant.

\section{References}

1. Aasa, A.: Precedences in specifications and implementations of programming languages. In: Selected Papers of the Symposium on Programming Language Implementation and Logic Programming, Elsevier Science Publishers B. V., Amsterdam, PLILP '91, pp. 3-26. http://dl.acm.org/citation. cfm?id=203429.203431 (1995)

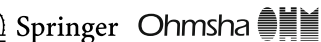

$\begin{array}{llll}\text { Journal : Small-ext 354 } & \text { Dispatch : } & \mathbf{1 3 - 2 - 2 0 2 0} & \text { Pages : } \mathbf{5 5} \\ \text { Article No. }: \mathbf{8 2} & \square \text { LE } & \end{array}$


2. Afroozeh, A., Izmaylova, A.: Faster, practical GLL parsing. In: Franke, B. (ed.) Compiler Construction, pp. 89-108. Springer, Berlin (2015). https://doi.org/10.1007/978-3-662-46663-6_5

3. Aho, A.V., Johnson, S.C., Ullman, J.D.: Deterministic parsing of ambiguous grammars. Commun. ACM 18(8), 441-452 (1975)

4. Appel, A.W.: Modern Compiler Implementation in ML, 1st edn. Cambridge University Press, New York (1998)

5. Bird, R.: Thinking Functionally with Haskell. Cambridge University Press, Cambridge (2014). https://doi.org/10.1017/CBO9781316092415

6. Boulton, R.: Syn: a single language for specifying abstract syntax trees, lexical analysis, parsing and pretty-printing. Tech. Rep. Number 390, Computer Laboratory, University of Cambridge (1966)

7. Brabrand, C., Møller, A., Schwartzbach, M.I.: Dual syntax for XML languages. Inf. Syst. 33(4-5), 385-406 (2008). https://doi.org/10.1016/j.is.2008.01.006

8. van den Brand, M., Visser, E.: Generation of formatters for context-free languages. ACM Trans. Softw. Eng. Methodol. 5(1), 1-41 (1996). https://doi.org/10.1145/226155.226156

9. van den Brand, M.G.J., Scheerder, J., Vinju, J.J., Visser, E.: Disambiguation filters for scannerless generalized LR parsers. In: Proceedings of the 11th International Conference on Compiler Construction, Springer, London, UK, CC '02, pp. 143-158. https://doi.org/10.1007/3-540-45937-5_12 (2002)

10. Cantor, D.G.: On the ambiguity problem of Backus systems. J. ACM 9(4), 477-479 (1962)

11. Czarnecki, K., Foster, J.N., Hu, Z., Lämmel, R., Schürr, A., Terwilliger, J.F.: Bidirectional transformations: a cross-discipline perspective. In: Proceedings of the 2nd International Conference on Theory and Practice of Model Transformations, Springer, Berlin, ICMT '09, pp. 260-283. https://doi. org/10.1007/978-3-642-02408-5_19 (2009)

12. Dijkstra, E.W.: Guarded commands, nondeterminacy and formal derivation of programs. Commun. ACM 18(8), 453-457 (1975). https://doi.org/10.1145/360933.360975

13. Duregård, J., Jansson, P.: Embedded parser generators. In: Proceedings of the 4th ACM Symposium on Haskell, ACM, New York, NY, USA, Haskell '11, pp. 107-117. https://doi.org/10.1145/2034675. 2034689 (2011)

14. Earley, J.: An efficient context-free parsing algorithm. Commun. ACM 13(2), 94-102 (1970). https:// doi.org/10.1145/362007.362035

15. Fischer, S., Hu, Z., Pacheco, H.: The essence of bidirectional programming. Sci. China Inf. Sci. 58(5), 1-21 (2015)

16. Foster, J.N.: Bidirectional programming languages. PhD thesis, University of Pennsylvania (2009)

17. Foster, J.N., Greenwald, M.B., Moore, J.T., Pierce, B.C., Schmitt, A.: Combinators for bidirectional tree transformations: a linguistic approach to the view-update problem. ACM Trans. Program. Lang. Syst. 29, 3 (2007). https://doi.org/10.1145/1232420.1232424

18. Fowler, M., Beck, K.: Refactoring: Improving the Design of Existing Code. Addison-Wesley Professional, Boston (1999)

19. Gibbons, J., Stevens, P.: International Summer School on Bidirectional Transformations (Oxford, UK, 25-29 July 2016). Lecture Notes in Computer Science, vol. 9715. Springer, Berlin (2018)

20. Gosling, J., Joy, B., Steele, G.: The Java Language Specification, 3rd ed (2006). https://docs.oracle. com/javase/specs/

21. Hirzel, M., Rose, K.H.: Tiger language specification (2013). https://cs.nyu.edu/courses/fall13/CSCIGA.2130-001/tiger-spec.pdf

22. Hu, Z., Ko, H.S.: Principles and practice of bidirectional programming in BiGUL. In: Gibbons, J., Stevens, P. (eds.) Bidirectional Transformations: International Summer School, Oxford, UK, July 25-29, 2016, Tutorial Lectures, pp. 100-150. Springer, Cham (2018). https://doi.org/10.1007/978-3319-79108-1_4

23. Johnson, S.C.: Yacc: Yet another compiler-compiler. AT\&T Bell Laboratories Technical Reports (AT\&T Bell Laboratories Murray Hill, New Jersey 07974). p. 32 (1975)

24. Kernighan, B.W., Ritchie, D.M.: The C Programming Language. Prentice Hall Press, Upper Saddle River (1988)

25. Kinoshita, D., Nakano, K.: Bidirectional certified programming. In: Eramo, R., Johnson. M. (eds) Proceedings of the 6th International Workshop on Bidirectional Transformations Co-Located with The European Joint Conferences on Theory and Practice of Software (ETAPS 2017), CEUR Workshop Proceedings, Uppsala, Sweden, vol. 1827, pp. 31-38 (2017)

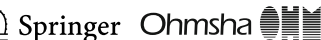

\begin{tabular}{|l|lllll|}
\hline & Journal : Small-ext 354 & Dispatch : $\mathbf{1 3 - 2 - 2 0 2 0}$ & Pages : 55 \\
& Article No. : 82 & $\square$ & LE & & TYPESET \\
& MS Code : NGCO-D-19-00023R2 & $\nabla$ & CP & $\square$ & DISK \\
\hline
\end{tabular}


26. Klint, P., Visser, E.: Using filters for the disambiguation of context-free grammars. In: Pighizzini, G., Pietro, P.S. (eds) Proceedings of the ASMICS Workshop on Parsing Theory, University of Milan, Italy, Milano, Italy, pp. 1-20 (1994)

27. Ko, H.S., Hu, Z.: An axiomatic basis for bidirectional programming. Proc. ACM Program. Lang. 2(POPL), 41:1-41:29 (2018). https://doi.org/10.1145/3158129

28. Ko, H.S., Zan, T., Hu, Z.: BiGUL: a formally verified core language for putback-based bidirectional programming. In: Proceedings of the 2016 ACM SIGPLAN Workshop on Partial Evaluation and Program Manipulation, ACM, New York, NY, USA, PEPM '16, pp. 61-72 (2016). https://doi.org/10. $1145 / 2847538.2847544$

29. LaLonde, W.R., des Rivieres, J.: Handling operator precedence in arithmetic expressions with tree transformations. ACM Trans. Program. Lang. Syst. 3(1), 83-103 (1981). https://doi.org/10.1145/ 357121.357127

30. Lämmel, R., Jones, S.P.: Scrap your boilerplate: a practical design pattern for generic programming. In: Proceedings of the 2003 ACM SIGPLAN International Workshop on Types in Languages Design and Implementation, ACM, New York, NY, USA, TLDI '03, pp. 26-37 (2003). https://doi.org/10. $1145 / 604174.604179$

31. Lutterkort, D.: Augeas-a configuration API. In: Proceedings of the Ottawa Linux Symposium, Ottawa, Canada, pp. 47-56 (2008)

32. Macedo, N., Pacheco, H., Cunha, A., Oliveira, J.N.: Composing least-change lenses. Proc. Sec. Int. Workshop Bidirect. Transform. 57, 1-19 (2013). https://doi.org/10.14279/tuj.eceasst.57.868

33. Marlow, S., Gill, A.: The parser generator for Haskell. https://www.haskell.org/happy/ (2001)

34. Marlow, S., et al.: Haskell 2010 language report. https://www.haskell.org/onlinereport/haskell2010/ (2010)

35. Martins, P., Saraiva, J., Fernandes, J.P., Van Wyk, E.: Generating attribute grammar-based bidirectional transformations from rewrite rules. In: Proceedings of the ACM SIGPLAN 2014 Workshop on Partial Evaluation and Program Manipulation, ACM, New York, NY, USA, PEPM '14, pp. 63-70 (2014). https://doi.org/10.1145/2543728.2543745

36. Matsuda, K., Wang, M.: Embedding invertible languages with binders: a case of the FliPpr language. In: Proceedings of the 11th ACM SIGPLAN International Symposium on Haskell, ACM, New York, NY, USA, Haskell 2018, pp. 158-171 (2018a). https://doi.org/10.1145/3242744.3242758

37. Matsuda, K., Wang, M.: FliPpr: a system for deriving parsers from pretty-printers. New Gener. Comput. 36(3), 173-202 (2018b). https://doi.org/10.1007/s00354-018-0033-7

38. Matsuda, K., Mu, S.C., Hu, Z., Takeichi, M.: A grammar-based approach to invertible programs. In: Gordon, A.D. (ed) Proceedings of the 19th European Conference on Programming Languages and Systems, Springer, Berlin, no. 20 in ESOP'10, pp. 448-467 (2010). https://doi.org/10.1007/978-3642-11957-6_24

39. Norell, U.: Towards a practical programming language based on dependent type theory. PhD thesis, Chalmers University of Technology (2007)

40. Pacheco, H., Hu, Z., Fischer, S.: Monadic combinators for "putback" style bidirectional programming. In: Proceedings of the ACM SIGPLAN 2014 Workshop on Partial Evaluation and Program Manipulation, ACM, New York, NY, USA, PEPM '14, pp. 39-50 (2014a). https://doi.org/10.1145/ 2543728.2543737

41. Pacheco, H., Zan, T., Hu, Z.: BiFluX: A bidirectional functional update language for XML. In: Proceedings of the 16th International Symposium on Principles and Practice of Declarative Programming, ACM, New York, NY, USA, PPDP '14, pp. 147-158 (2014b). https://doi.org/10.1145/ 2643135.2643141

42. Pombrio, J., Krishnamurthi, S.: Resugaring: lifting evaluation sequences through syntactic sugar. In: Proceedings of the 35th ACM SIGPLAN Conference on Programming Language Design and Implementation, ACM, New York, NY, USA, no. 6 in PLDI '14, pp. 361-371 (2014). https://doi.org/ $10.1145 / 2594291.2594319$

43. Pombrio, J., Krishnamurthi, S.: Hygienic resugaring of compositional desugaring. In: Proceedings of the 20th ACM SIGPLAN International Conference on Functional Programming, ACM, New York, NY, USA, no. 13 in ICFP 2015, pp. 75-87 (2015). https://doi.org/10.1145/2784731.2784755

44. Rendel, T., Ostermann, K.: Invertible syntax descriptions: unifying parsing and pretty printing. In: Proceedings of the Third ACM Haskell Symposium on Haskell, ACM, New York, NY, USA, Haskell '10, pp. 1-12 (2010). https://doi.org/10.1145/1863523.1863525

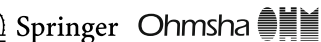

\begin{tabular}{|l|lllll|}
\hline & Journal : Small-ext 354 & Dispatch : 13-2-2020 & Pages : 55 \\
Article No. : 82 & $\square$ LE & $\square$ & TYPESET \\
& MS Code : NGCO-D-19-00023R2 & $\square$ CP & $\square$ & DISK \\
\hline
\end{tabular}


1653

1654

1655

1656

1657

1658

1659

1660

1661

1662

1663

1664

1665

1666

1667

1668

1669

1670

1671

1672

1673

1674

1675

1676

1677

1678

1679

1680

1681

1682

1683

1684

\section{Affiliations} 357218

$\triangle$ Zirun Zhu

zhu@nii.ac.jp

Pedro Martins

João Saraiva

Zhenjiang $\mathrm{Hu}$

45. Reps, T., Teitelbaum, T.: The synthesizer generator. In: Proceedings of the First ACM SIGSOFT/ SIGPLAN Software Engineering Symposium on Practical Software Development Environments, ACM, New York, NY, USA, SDE 1, pp. 42-48 (1984). https://doi.org/10.1145/800020.808247

46. Reps, T., Teitelbaum, T., Demers, A.: Incremental context-dependent analysis for language-based editors. ACM Trans. Program. Lang. Syst. 5(3), 449-477 (1983). https://doi.org/10.1145/2166.

47. Scott, E., Johnstone, A.: GLL parsing. Electron. Notes Theor. Comput. Sci. 253(7), 177-189 (2010). https://doi.org/10.1016/j.entcs.2010.08.041

48. Scott, E., Johnstone, A., Economopoulos, R.: BRNGLR: a cubic tomita-style GLR parsing algorithm. Acta Inform. 44(6), 427-461 (2007). https://doi.org/10.1007/s00236-007-0054-Z

49. Sheard, T., Jones, S.P.: Template meta-programming for Haskell. In: Proceedings of the 2002 ACM SIGPLAN Workshop on Haskell, ACM, New York, NY, USA, Haskell '02, pp. 1-16 (2002). https:// doi.org/10.1145/581690.581691

50. Tomita, M.: An efficient context-free parsing algorithm for natural languages. In: Proceedings of the 9th International Joint Conference on Artificial Intelligence-Volume 2, Morgan Kaufmann Publishers Inc., San Francisco, CA, USA, IJCAI'85, pp. 756-764 (1985). http://dl.acm.org/citation.cfm?id= 1623611.1623625

51. Traver, V.J.: On compiler error messages: what they say and what they mean. Ad. Hum. Comput. Interact. 2010, 3:1-3:26 (2010). https://doi.org/10.1155/2010/602570

52. Visser, E.: A case study in optimizing parsing schemata by disambiguation filters. International Workshop on Parsing Technology (IWPT 1997), pp. 210-224. Massachusetts Institute of Technology, Boston, USA (1997a)

53. Visser, E.: Syntax definition for language prototyping. PhD thesis, University of Amsterdam (1997b)

54. Younger, D.H.: Recognition and parsing of context-free languages in time $n^{3}$. Inf. Control 10(2), 189-208 (1967)

55. Zhu, Z., Zhang, Y., Ko, H.S., Martins, P., Saraiva, J., Hu, Z.: Parsing and reflective printing, bidirectionally. In: Proceedings of the 2016 ACM SIGPLAN International Conference on Software Language Engineering, ACM, New York, NY, USA, SLE 2016, pp. 2-14. https://doi.org/10.1145/ 2997364.2997369 (2016)

Publisher's Note Springer Nature remains neutral with regard to jurisdictional claims in published maps and institutional affiliations.

\section{Zirun Zhu ${ }^{1}$ (1) $\cdot$ Hsiang-Shang Ko ${ }^{2}$ (1) $\cdot$ Yongzhe Zhang $^{1}$ (D) $\cdot$ Pedro Martins ${ }^{3} \cdot$ João Saraiva ${ }^{4}(1) \cdot{\text { Zhenjiang } \mathrm{Hu}^{5}}^{\circ}$}

Hsiang-Shang Ko

joshko@iis.sinica.edu.tw

Yongzhe Zhang

zyz915@nii.ac.jp

pribeiro@uci.edu

jas@di.uminho.pt

huzj@pku.edu.cn

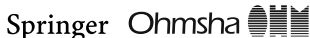

\begin{tabular}{|l|llll|}
\hline & Journal : Small-ext 354 & Dispatch : & $\mathbf{1 3 - 2 - 2 0 2 0}$ & Pages : $\mathbf{5 5}$ \\
Article No. : 82 & $\square \quad$ LE & $\square$ & TYPESET \\
\hline
\end{tabular}


$1701 \quad 1$ National Institute of Informatics, Chiyoda, Tokyo, Japan

17022 Institute of Information Science, Academia Sinica, Taipei, Taiwan

$17033^{3}$ University of California, Irvine, USA

17044 Department of Informatics, University of Minho, Braga, Portugal

17055 Department of Computer Science and Technology, Peking University, Beijing, China 